\title{
Rursus
}

Russus

Poiétique, réception et réécriture des textes antiques

$11 \mid 2017$

Nature et morale : sources, et postérité homilétique, des encyclopédies du XIIle siècle

\section{La production encyclopédique de Marc d'Orvieto et Juan Gil de Zamora : ressources pour la prédication}

Marcus of Orvieto's and Juan Gil of Zamora's encyclopedic Production:

Resources for Preaching

Adrienne Hamy-Dupont

\section{OpenEdition}

Journals

Édition électronique

URL : http://journals.openedition.org/rursus/1369

DOI : $10.4000 /$ rursus. 1369

ISSN : 1951-669X

Éditeur

Université Nice-Sophia Antipolis

Référence électronique

Adrienne Hamy-Dupont, « La production encyclopédique de Marc d'Orvieto et Juan Gil de Zamora ressources pour la prédication », Rursus [En ligne], 11 | 2017, mis en ligne le 20 octobre 2017, consulté le 21 avril 2019. URL : http://journals.openedition.org/rursus/1369; DOI : 10.4000/rursus.1369

Ce document a été généré automatiquement le 21 avril 2019

Rursus 


\title{
La production encyclopédique de Marc d'Orvieto et Juan Gil de Zamora : ressources pour la prédication
}

\author{
Marcus of Orvieto's and Juan Gil of Zamora's encyclopedic Production: \\ Resources for Preaching
}

Adrienne Hamy-Dupont

\section{Le sermon inséré dans la matière encyclopédique : un procédé original ?}

1 En travaillant sur les moralisations que le polygraphe Juan Gil de Zamora a greffées sur la matière encyclopédique de son Historia naturalis ${ }^{1}$, j'y ai relevé une panoplie complète de matériaux susceptibles d'entrer dans la composition d'un sermon'2, incluant la mise en forme la plus achevée: le sermon lui-même. De type "universitaire», c'est-à-dire construit sur un verset thématique selon la forme moderne qui s'impose au XIII siècle, une douzaine de sermons complets se trouvent insérée dans divers développements ${ }^{3}$ : 148-164 De abisso, 216-226 De accipitre, 274-280 De agno, 744-750 De apibus, 922-924 De aqua, 934-938 De aquila, 1012-1016 De archu celesti, 1026-1030 De argento, 1530-1534 De auro, 1536-1538 De austro, parfois doublement moralisés. L'abeille par exemple est comparée à sainte Cécile puis à saint Ambroise, l'abîme à saint Thomas puis au Christ.

Ce type d'insertion est-il original ? Les moralisations avaient-elles déjà leur place dans le discours encyclopédique «d'origine »: chez Thomas de Cantimpré4 ${ }^{4}$, Barthélemy l'Anglais ou Vincent de Beauvais ${ }^{5}$ Plus tardif, Jean de San Gimignano ${ }^{6}$ emploie-t-il les mêmes procédés? Une première approche comparative des générations successives de « naturalistes » du XIII ${ }^{\mathrm{e}}$ siècle m'a permis de relever d'intéressants échos dans l'usage des sources et méthodes homilétiques chez Juan Gil de Zamora et chez Marc d'Orvieto, 
possible auteur du Liber de moralitatibus. Ce dernier est, dans l'actuel état de la recherche, le plus voisin de mon objet.

3 Juan Gil et Marc d'Orvieto tissent tous deux, de manière assez similaire, des liens entre information encyclopédique et prédication. Mais le rapport établi est-il direct? Et les outils utilisés sont-ils nouveaux ?

\section{Des moralisations : définition}

4 La moralisation des quaestiones naturales est un procédé polymorphe dont la fécondité n'a pas été complètement sondée par la recherche : ces dernières décennies voient fleurir les études sur les réécritures des encyclopédies du premier XIII ${ }^{\mathrm{e}}$ siècle ${ }^{8}$, "genre " noncanonique et très variable par nature ${ }^{9}$. Ses manifestations en effet font encore l'objet de découvertes aux riches incidences: ainsi des marginalia d'ordre moral jusqu'il y a peu ignorés des éditeurs ${ }^{10}$, ainsi de l'insertion de sermons complets au cœur des notices encyclopédiques. La première a donné lieu pour Barthélemy l'Anglais, à des étude récentes ${ }^{11}$. La seconde est l'objet de ce travail pour un auteur particulier, Juan Gil de Zamora $^{12}$.

5 Comment reconnaître une moralisation? Dans les marges ou intégrée au texte ${ }^{13}$, elle accompagne la description des caractéristiques d'un élément naturel, dont elle procure une explication morale voire mystique selon une méthode souvent allégorique ou tropologique. Démarche de science, démarche de foi, elle constitue une interprétation spirituelle des propriétés naturelles. Recourant parfois aux Écritures et à des sources scientifiques et théologiques variées dans son état développé, elle est un précieux outil pour le prédicateur, aux côtés des florilèges, dictionnaires et concordances qui se multiplient au XIII ${ }^{e}$ siècle $^{14}$. Elle élabore l'herméneutique d'une Création divine décrite, classifiée, contemplée, destinée à louer le Créateur, mener une vie bonne et, pastoralement, permettre une prédication efficace, adaptée à une diversité de publics.

\section{Les auteurs en jeu et le corpus considéré}

6 Juan Gil et Marc sont des compilateurs appartenant tous deux à l'ordre des Frères mineurs, tout comme l'un de leurs modèles principaux, Barthélemy l'Anglais, dont le De proprietatibus rerum a été rédigé vers $1240^{15}$.

7 Leur biographie et notamment leurs années de formation, probablement parisiennes, comportent encore bien des mystères. Il est depuis peu avéré que le franciscain Juan Gil fut vicaire de sa province de Compostelle, ou du Portugal, en $1292^{16}$. Marc, s'il est bien l'auteur du Liber de moralitatibus, l'a probablement rédigé dans les années quatre-vingt du même siècle, datation qui se fonde sur la dédicace à Benedetto Caetani, cardinal diacre de San Nicola in Carcere depuis 1281, futur pape sous le nom de Boniface viII dès $1291^{17}$. Juan Gil et Marc ont donc été actifs dans les mêmes décennies et s'inscrivent tous deux dans ce que l'on pourrait qualifier de deuxième génération de "naturalistes", producteurs d'encyclopédies moralisées ${ }^{18}$.

8 Les deux œuvres que j'examinerai sont d'une part l'Historia naturalis (= HN) de Juan Gil, d'autre part le Liber de moralitatibus (= LM) ou Liber septiformis ou De proprietatibus moralizatus de Marc. J'y joindrai des comparaisons ponctuelles avec le corpus de marginalia 
du De proprietatibus rerum (= PR) de Barthélemy l'Anglais, dont l'édition critique est en cours dans la collection De diversis artibus ${ }^{19}$.

S'il ne reste de la première œuvre (HN) que la lettre A, peut-être la seule traitée par Juan $\mathrm{Gil}^{20}$, les trois volumes de son édition par García Ballester n'en sont pas moins copieux, de l'asbeste à l'azurite, notices transmises par deux manuscrits (Ms. Berlin, Staatsbibliothek, Lat. Fol. 62 et Ms. Escorial, P.I.10) et complétées par un traité spécialisé De animalibus transmis par le seul manuscrit Escorial ${ }^{21}$. Quant au LM, il est qualifié par Etzkorn, son éditeur, de "moralisation» du De proprietatibus rerum de Barthélémy l'Anglais et de représentant de la littérature d'exempla. Il faudrait nuancer cette proposition, car Marc, son auteur présumé, met évidemment à contribution des autorités scientifiques bien plus diverses que le seul Barthélemy; en outre, plus que du recueil exemplaire, le texte se rapproche des distinctiones.

L'HN propose un classement intégralement alphabétique de la matière ${ }^{22}$, traitant ainsi de l'adriana avant l'angelus. Juan Gil adopte d'ailleurs ce même ordo artificialis dans son Archivus sive Armarium scripturarum, son Liber illustrium personarum, dans les chapitres XII, XIII et XV de son Prosodion, les oraciones du livre XVIII de son Liber Marie, dans son Liber contra uenena et ses Legende sanctorum ${ }^{23}$. L'auteur du LM, lui, emploie un système alphabétique au sein seulement des parties de son ouvrage hexaméral dont voici les

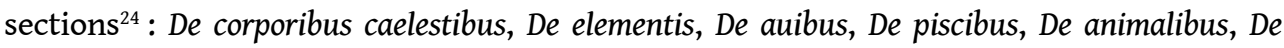
arboribus et plantis, De lapidibus pretiosis.

\section{Procédés homilétiques}

11 Les notices de Marc s'apparentent davantage à un recueil de distinctiones qu'à un catalogue d'exempla, ai-je suggéré. Les distinctiones en effet sont des répertoires d'éléments bibliques et de commentaires patristiques classés dans l'ordre des livres de l'Écriture puis par ordre alphabétique des mots vedettes ${ }^{25}$. Outil scolaire à la fin du XII ${ }^{\mathrm{e}}$ siècle, ces ouvrages deviennent ensuite une ressource pour les prédicateurs ${ }^{26}$. Dans le LM, ce sont les éléments naturels qui sont classés par catégories puis par ordre alphabétique. La description de leurs propriétés est suivie de passages bibliques, de commentaires patristiques et des diverses interprétations données par l'exégèse et sélectionnées par concordance verbale ou thématique. Pourrait-on postuler qu'il s'agit chez Marc de distinctiones non plus bibliques mais encyclopédiques?

Juan Gil, lui, use d'une palette plus grande pour illustrer les moralisations qui viennent parfois enrichir ses notices naturelles: méditations, similitudines simples ou multiples, proches en ce cas de la distinctio voire du schéma homilétique, allant jusqu'aux sermons complets.

13 Dresser un parallèle de ces deux auteurs avec l'une de leurs sources principales, Barthélemy l'Anglais ${ }^{27}$, s'est rapidement imposé. Avec déception tout d'abord. Paradoxalement, le PR présente un texte fort peu allégorique, lui qui pourtant affichait dès le prologue son intention de décrypter les Écritures "grâce à l'explicitation des symboles et des figures des choses naturelles et artificielles qui y sont présentes »:

Vtile mihi et forsitan aliis, qui naturas rerum et proprietates per sanctorum libros necnon et philosophorum dispersas non cognoverunt, ad intelligenda enigmata scripturarum, que sub symbolis et figuris proprietatum rerum naturalium et artificialium a Spiritu sancto sunt tradite et velate. (BARTHOLOMAEUS ANGLICUS, De proprietatibus rerum, Proemium) ${ }^{28}$ 
C'est en réalité vers les marges de certains de ses témoins qu'il faut se tourner. Un vaste corpus de notes morales (de neuf à dix mille occurrences par témoin) y est élaboré, très tôt dans la tradition manuscrite, voire du vivant de Barthélemy ou par Barthélemy lui-même, si l'on adopte l'hypothèse la plus audacieuse ${ }^{29}$. Il s'agit de brèves phrases nominales signalant la portée moralisante ou allégorique de certains passages. Cet apparat marginal débute au chapitre cinq du quatrième livre, consacré au corps humain ${ }^{30}$. Des travaux menés en collaboration à Münster et à Louvain-la-Neuve ${ }^{31}$ ont permis de déterminer que ces notes ne sont pas un épiphénomène de la tradition manuscrite mais bien un corpus cohérent en marge de la plupart des manuscrits des XIII ${ }^{\mathrm{e}}$ et XIV ${ }^{\mathrm{e}}$ siècle. C'est pourquoi les éditeurs de l'édition critique en cours ont résolu de les y intégrer ${ }^{32}$. Ainsi, chez Barthélemy, les notes morales accompagnant le cours de la notice De sapphiro sont les suivantes dans le ms. Paris, Bibliothèque Nationale de France, lat. 16099, fol. 137vab ${ }^{33}$ :

Nota de uirgine benedicta. Nota de uirtutibus occultis uirginis. Nota quod ipsa est regina omnium. Nota de eius humilitate. Nota de maria magdalena et eius penitentia. Nota de maria matre dei. Nota de humanitate christi. Nota quod maria dei et hominum est reconciliatrix. Nota quod maria extinguit in nobis estum concupiscencie. Nota quod maria est letitie spiritualis causa. Nota de uirtute contra concupiscentiam. Nota de humilitate uirginali. Nota contra maliciam diaboli et peccati. Nota quod maria cunctas hereses interimit. Nota quod omnia mala a corde tollit. Nota quod per mariam omnes preces exaudiuntur apud deum. Nota quod per mariam soluuntur uincula peccatorum. Nota de castitate. Nota contra inuidiam et timorem. (BARTHOLOMAEUS ANGLICUS, PR : 16.87).

Quant aux auteurs de l'HN et du LM, lorsqu'ils complètent leurs notices au moyen d'équivalences exégétiques, par exemple la pénitence ou le péché mortel pour la plante absinthe dont il est question ci-dessous, dévoilent-ils par là une part plus personnelle de leur ouvrage?

Absinthio autem, propter sui amaritudinem, potest penitencia comparari (IOHANNES AEGIDII ZAMORENSIS, HN : 144), et :

Absinthus has habet condiciones (...) Significat peccatum mortale cum suis effectibus (MARCUS URBEVETENSIS, Liber de Moralitatibus : 831).

17 Plus certainement, ces équivalences ont elles-mêmes une source et sont le signe de l'usage de manuscrits annotés de marginalia moraux. Un travail de comparaison systématique de l'HN avec les notices du LM et les marginalia du PR reste à mener lorsque ces dernières seront toutes disponibles.

Le tableau suivant présente un aperçu de ce que j'ai pu découvrir pour le sapin, De abiete arbore. Juan Gil comme Marc empruntent pour cette notice leurs développements scientifiques (non retranscrits ici) à Barthélemy. Ils lui empruntent aussi ses moralisations, mais seulement partiellement, en respectant l'ordre et la formulation, comme on le voit dans le tableau ci-dessous. Trois moralisations de Barthélemy sont communes à Marc et Juan Gil: de religiosis, de paupertate, de rectitudine iustitie. Marc reprend six marginalia supplémentaires: de humilitate, de operatione caritatis, de utilitate religiosorum. 


\begin{tabular}{|c|c|c|}
\hline $\begin{array}{l}\text { BARTHOLOMAEUS } \\
\text { ANGLICUS } \\
\text { PR : } \mathbf{1 7 . 4} \\
\text { Marginalia }^{34}\end{array}$ & $\begin{array}{l}\text { IOHANNES AEGIDII ZAMORENSIS } \\
\text { HN } \mathbf{1 . 2}: \mathbf{1 2 8 - 1 3 0}\end{array}$ & $\begin{array}{l}\text { MARCUS URBEVETENSIS } \\
\text { LM } 6.3 \mathbf{~ : ~ 8 0 2 - 8 0 3}\end{array}$ \\
\hline $\begin{array}{l}\text { Nota de } \\
\text { religiosis et } \\
\text { contemplativis }\end{array}$ & $\begin{array}{l}\text { (...) Sicut autem abies est expers } \\
\text { terreni humoris }{ }^{35} \text {,, ita et status } \\
\text { religionis. }\end{array}$ & $\begin{array}{l}\text { (...) abies dicitur ab eundo, eo quod } \\
\text { plus ceteris arboribus a terra in altum } \\
\text { altius se expandit }{ }^{36} \text {. Significat } \\
\text { religiosos et contemplatiuos plus } \\
\text { ceteris in Deum ascendentes et diuinis } \\
\text { se supermentaliter expandentes (...) }\end{array}$ \\
\hline \multirow[t]{3}{*}{$\begin{array}{l}\text { Nota } d \epsilon \\
\text { paupertate } \\
\text { religiosorum }\end{array}$} & $\begin{array}{l}\text { Et sicut abies habet plurimum } \\
\text { raritatis }^{37} \text {, ita et status paupertatis. }\end{array}$ & $\begin{array}{l}\text { (...) arbor haec est expers terreni } \\
\text { humoris, et ideo habilis atque leuis }{ }^{38} \text {. } \\
\text { Significat pauperes rebus uoluntaria } \\
\text { deuotione agiles esse ad sursum } \\
\text { actiones mentales (...) }\end{array}$ \\
\hline & $\begin{array}{l}\text { Et sicut in abiete reperitur equalitas, } \\
\text { ita et in iusto iudice iudicii equitas. }\end{array}$ & \\
\hline & $\begin{array}{l}\text { Sicut eciam abies propter sui } \\
\text { unctuositatem et gumositatem ab } \\
\text { igne materiali faciliter inflammatur, } \\
\text { ita et anima, devocionis gracia } \\
\text { impinguata, mirabiliter inflammatur, } \\
\text { cum eidem ignitum divinum } \\
\text { eloquium predicant. }\end{array}$ & \\
\hline $\begin{array}{l}\text { Nota } \\
\text { humilitate }\end{array}$ & & $\begin{array}{l}\text { (...) arbor haec habet plurimum } \\
\text { raritatis, substantiaque eius est } \\
\text { subtilis humiditatis, et ideo calor eius } \\
\text { naturalis a solis calore fortificatus, } \\
\text { illam humiditatem eleuat de facili et } \\
\text { per sursum nutrit et mittit, et sic } \\
\text { ipsam in arboris substantiam } \\
\text { conuertens, arborem ipsam altam facit } \\
\text { 39. Significat in uiro bono humilitatem } \\
\text { esse uiam ad amorem Dei et } \\
\text { celsitudinem meriti (...) }\end{array}$ \\
\hline $\begin{array}{l}\text { Nota de } \\
\text { fortitudine } \\
\text { caritatis }\end{array}$ & & \\
\hline
\end{tabular}




\begin{tabular}{|c|c|c|}
\hline $\begin{array}{ll}\text { Nota de } & \text { de } \\
\text { rectitudine } & \\
\text { iustitie } & \end{array}$ & $\begin{array}{l}\text { Et sicut abies in structuris domorum } \\
\text { materialium est utilis propter suam } \\
\text { rectitudinem, quia parvam vel } \\
\text { nullam habet obliquitatem, ita et } \\
\text { anima in rectitudine iusticie fundata } \\
\text { neccessaria et utilis semper est ad } \\
\text { construendam causarum } \\
\text { spiritualium veritatem. }\end{array}$ & $\begin{array}{l}\text { (...) est arbor mirae rectitudinis, et } \\
\text { quasi nihil habens tortuositatis }{ }^{40} \text {. } \\
\text { Significat amatores ueritatis et } \\
\text { iustitiae pro caelesti intentione (...) }\end{array}$ \\
\hline & $\begin{array}{l}\text { Et sicut abies velificacioni navium } \\
\text { aptatur, ita et anima sancti viri per } \\
\text { pacienciam armatur ad tolerandum } \\
\text { fluctus persecucionum temporalium } \\
\text { pacienter. Malus enim fit ex abiete et } \\
\text { ei velum apponitur, quo navis } \\
\text { ventorum impetu agitatur et } \\
\text { dirigitur usque ad portum, ita et } \\
\text { rectitudo anime, per velum paciencie } \\
\text { appositum, dum tribulacionibus } \\
\text { seculi concutitur, ad portum dirigitur } \\
\text { salutarem. }\end{array}$ & \\
\hline & & $\begin{array}{l}\text { (...) quamuis haec arbor proceretur ex } \\
\text { humore leui et subtili, illius tamen } \\
\text { humoris superfluitatem reicit. Nam ad } \\
\text { eius superficiem, scilicet inter lignum } \\
\text { et corticem, et ibidem a calore } \\
\text { interiori inuiscatur, et in naturam } \\
\text { cuiusdam resinae odoriferae } \\
\text { transmutatur }{ }^{41} \text {. Significat bonos uiros } \\
\text { facientes sibi de necessitate uirtutem, } \\
\text { et omnia etiam fragilia sapienter } \\
\text { ordinantes ad aliorum aedificationem } \\
\text { (...) }\end{array}$ \\
\hline $\begin{array}{l}\text { Nota de effectu } \\
\text { et operatione } \\
\text { caritatis }\end{array}$ & & $\begin{array}{l}\text { (...) propter suam caliditatem igni } \\
\text { apposita facillime inflammatur. } \\
\text { Significat homines caritatiuos cito } \\
\text { affici semper ad aspectum afflictorum } \\
\text { siue infirmantium uel alias } \\
\text { miserabilium personarum (...) }\end{array}$ \\
\hline $\begin{array}{ll}\text { Nota de } \\
\text { compassione et } \\
\text { pietate }\end{array}$ & & \\
\hline
\end{tabular}




\begin{tabular}{|c|c|}
\hline $\begin{array}{l}\text { Nota de } \\
\text { utilitate } \\
\text { religiosorum }\end{array}$ & $\begin{array}{l}\text { (...) uariis aedificiorum structuris haec } \\
\text { arbor accomodatur, et maxime } \\
\text { propter suam rectam altitudinem et } \\
\text { formam pyramidalem adaptationis } \\
\text { nauium utiliter adaptatur }{ }^{42} \text {. Significat } \\
\text { utilitatem uariam et multiformem } \\
\text { quam faciunt religiones in Ecclesia Dei } \\
\text { et animabus diuersis siue populis } \\
\text { propter suorum meritorum et } \\
\text { documentorum } \\
\text { euidentem (...) }\end{array}$ \\
\hline
\end{tabular}

19 Le travail est archéologique : Marc et Juan Gil se sont-ils inspirés de témoins manuscrits annotés du PR? C'est plus que probable, même si ces deux auteurs ont pu recourir, comme Barthélemy, à des compendia plus anciens de caractéristiques naturelles destinés à l'exégèse. Ces compendia sont des lexiques mettant en rapport allégorique les propriétés du créé : le Vocabularium de Papias, le dictionnaire d'Uguccio de Pise, et des recueils de propriétés utilisés dès la fin du XII ${ }^{\mathrm{e}}$ siècle : l'« Anonyme de Clairvaux ", ou l'Angelus purus natura $a^{43}$.

Le lecteur médiéval - prédicateur, naturaliste, érudit -, en parcourant les marges des manuscrits concernés du De proprietatibus rerum de Barthélemy l'Anglais, pouvait rapidement y récolter les données nécessaires à l'élaboration d'un sermon ou traité, sur la chasteté par exemple. Ainsi, parmi nombre d'autres éléments naturels, la laitue, la tortue, le saphir, la poule, la terre, le cèdre, l'ébène ont, dans les marginalia de Barthélemy, la chasteté pour moralisation.

Quant au procédé employé par Marc d'Orvieto, il peut équivaloir à une sorte de modèle de sermon «à choix multiples ». En effet, à un élément naturel brièvement présenté sont associées diverses caractéristiques en nombre variable, de deux à quarante-deux. Ces caractéristiques peuvent constituer autant de parties possibles d'un sermon. Car chacune appelle des auctoritates explicites: scientifiques, bibliques et théologiques par le biais d'une similitudo généralement introduite par le verbe significare. Au sein du LM, le prédicateur pressé peut donc trouver une interprétation morale des mystères de la Création divine sous forme de parties de sermons détachables et prêtes-à-porter. Le sermon dit "moderne ", « universitaire ", "scolastique » ou encore " thématique » ${ }^{44}$ est fils des divisions et subdivisions qui règnent en maître dans les sommes et quaestiones contemporaines. En voici un exemple pour la notice du LM sur le saphir, dont je donne la première et la dernière significatio, explicitées entre crochets :

Sapphirus, qui est quintus pretiosus lapis in rationali Aaron, Exod. 39 (39.11), et septimus in operimento primi anguli, Ez. 28 (28.13), has habet condiciones siue proprietates.

[Classification :] Primo enim, [Auctoritates scientifiques :] ut ponit Isidorus et Dioscorides et habetur in Lapidario, [Proprietas:] dicitur gemma gemmarum. [ Significatio :] Significat beatam Virginem propter excellentiam gratiae et gloriae respectu aliorum sanctorum et angelorum omnium, [Auctoritas biblique :] qua sola inter mulieres gratia plena et benedicta, Luc. 1 (1.42), describitur. [Auctoritates patristique et liturgique :] Nam secundum Hieronymum: "Gratia ceteris per partes praestatur, Mariae uero se totam infudit gratiae plenitudo et diuinitatis 
unda ». Vnde et merito super choros angelorum exaltata cantatur, et a beato Hieronymo in sermone De assumptione praedicatur. Et

[Classification :] Decimo quinto, [Auctoritas scientifique :] ut dicit Dioscorides, [ Proprietas :] uim habet ulcerum curatiuam. [Similitudo :] Significat humilitatem euangelicam omnia spiritualia uulnera et passiones animae propellentem, [ Auctoritas biblique :] Matth. 11 (11.29): Discite a me etc. et inuenietis requiem animabus uestris. [Auctoritas patristique :] Augustinus De sancta uirginitate: « Non enim uenitur aliter requies animae nisi in quieto tumore digesto ». (MARCUS URBEVETENSIS, LM 7.82).

Cette divisio par rationes se retrouve également chez Juan Gil de Zamora, témoin le début de la moralisation de la notice De abisso. L'abîme y est comparé aussi bien à Dieu qu'à l'enfer, à un avare, à la bonté divine, à saint Thomas et au Christ. Au lecteur d'élire les objets de comparaison proposés pour bâtir un sermon, entamer une méditation ou encore construire un développement encyclopédique :

\footnotetext{
Abissus itaque ex se habet obscuritatem, profunditatem, omnium aquarum et fluidorum capacitatem, insaciabilitatem, quia nunquam tot aquas recipit quin plures recipere possit, inscrutabilitatem, inhauribilitatem, communicabilitatem. [ Similitudo 1] Abisso Deus altissimus comparatur propter sui impenetrabilitatem. [ Similitudo 2] Infernus et peccator comparantur abisso propter sui obscuritatem. [ Similitudo 3] Avarus comparatur abisso propter sui insaciabilitatem. [Similitudo 4] Divina bonitas et pietas comparantur abisso propter sui communicabilitatem. [ Similitudo 5] Beatus Thomas specialiter comparatur abisso propter dubitacionis obscuritatem. [Similitudo 6] Vnde de Christo et de ipso merito dici potest quod abissus abissum invocet cataractum suarum (Ps. 41.8), id est, plagarum suarum, cum dixit ei : Vide manus meas et mitte digittum tuum in latus meum. Et noli esse incredulus, sed fidelis (Joh. 20.27). (IOHANNES AEGIDIIS ZAMORENSIS, HN : 148)
}

Les deux dernières comparaisons choisies par Juan Gil (le Christ et Thomas) donnent lieu, dans la suite de cette moralisation, à deux développements formant chacun un sermon en trois parties.

\section{Le sermon inséré dans le grand-œuvre de Juan Gil de Zamora}

L'avantage d'un ouvrage comme le LM est de proposer une classification de la materia praedicabilis par éléments naturels. Mais reste encore à l'utilisateur à bâtir son sermon, très codifié en cette fin de siècle : autour d'une citation biblique - le thema, divisé ensuite en autant d'éléments qu'il possède de mots-clefs - s'élabore une declaratio partium, puis s'échelonnent les diverses parties, généralement de deux à cinq. Ces dernières jouent des correspondances verbales ou thématiques fournies par les concordances bibliques et les florilèges. Elles proposent ainsi une dilatatio thematis par jeux d'échos et se clôturent par une doxologie.

Différente du commentaire patristique et monastique d'une péricope entière, c'est cette forme moderne du sermon thématique, nourrie d'un savoir exégétique fondé sur la distinction, qu'adjoint Juan Gil à certaines rubriques de son HN. Je n'ai, dans l'état actuel des connaissances, pas retrouvé les sermons de l'HN dans d'autres œuvres du polygraphe, pourtant adepte $\mathrm{du}$ « recyclage » de ses propres écrits ${ }^{45}$. 
Juan Gil de Zamora a composé un Opus sermonum copiosum, perdu, dont des sections sont partiellement disséminées dans divers recueils, homilétiques ${ }^{46}$ et théologiques. Ainsi, deux de ses ouvrages théologiques : son Liber Marie ${ }^{47}$, objet d'une thèse non publiée ${ }^{48}$, et son Liber Iesu ${ }^{49}$, encore totalement inédit, livrent des sermons, dont trois se retrouvent dans le sermonnaire d'Assise, Sacro Convento, 414. Ce dernier témoin contient quatrevingt cinq sermons distribués en deux collections: l'une pour diverses occasions ( temporales ${ }^{50}$, virginales ${ }^{51}$, ad status ${ }^{52}$ et rituales), l'autre intitulée Breviloquium de vitiis et virtutibus ${ }^{53}$.

8 Il est intéressant par ailleurs de noter que les sermons dissimulés dans le Liber Marie de Gil découlent d'un thema issu du monde naturel qui les informe - plantes, métaux, objets célestes - ou d'un thema conduisant par concordance thématique à un développement sur les res naturales ${ }^{54}$ : Orietur stella ex Iacob. Consurget uirga de Israel et percutiet duces Moab (Num. 24.17), Lux orta est iusto et rectis corde letitia (Ps. 96.11), Fons egreditur de domo Domini et irrigabit torrentem spinarum (Joel 3.18), Vas admirabile, opus Excelsi (Sir. 43.2), Dominus in celo parauit sedem suam (Ps. 102.19), In sole posuit Filius Dei tabernaculum suum (Ps. 19.6), Altissimus de terra creauit medicinam et uir sapiens non abhorrebit eam (Sir. 38.4), Venit lumen tuum Hierusalem (Is. 60.1), Positus est tronus matris regis et sedit ad dexteram eius (I Reg. 2.19), Quasi cedrus exaltata sum in Libano (Sir. 24.17).

Il est évident que ce procédé d'insertion de sermons dans des œuvres dont la finalité n'est pas immédiatement homilétique est courant chez Juan Gil. Il signe par là, semble-t-il, une volonté de mettre son grand-œuvre au service de la prédication qui couronne ainsi toute son entreprise. Dans sa vaste encyclopédie naturelle, l'Historia naturalis, Juan Gil introduit ces structures de sermons à la suite de l'exposition des propriétés d'un élément naturel. Comment procède-t-il ? Peu ou prou comme Marc, mais il délivre parfois des sermons intégraux, comprenant thema et doxologie et proposant deux à quatre parties. Pourquoi ne moralise-t-il pas systématiquement le contenu proposé ? Il répond lui-même à la question de la manière suivante, à la suite de sa notice sur la calvitie :

Per hec que dicta sunt multa ethyca patent, et ideo sufficit dare viam in aliquibus, non in omnibus, propter prolixitatem nimiam evitandam (IOHANNES AEGIDII ZAMORENSIS, HN : 362 ).

Un exemple particulièrement complet et complexe de sermon est inséré par Juan Gil dans son $\mathrm{HN}$ à la suite d'une notice sur l'or. Le lemme thématique, tiré de Job 28.6, met à contribution l'or et le saphir, qui sont comparés à la vie de saint Jacques et à ses miracles post mortem. En voici les principales articulations, explicitées entre crochets :

Aurum ab « aura » est dictum, ut dicit Hysidorus (...). [Similitudo :] Auro potest vita beati Iacobi comparari, [Thema:] et idcirco in ipsius preconium potest summi verbum illud quod scribitur Iob vigessimo octavo: Locus saphiri lapides eius et glebe illius aurum (Job 28.6). [Declaratio partium :] Graciosus et speciosus in sua conversacione ante mortem, gloriosus et miraculosus in sui visitacione post mortem. [Divisio:] Locus saphiri lapides: sepulcrum eius saphirus, propter multiplicem utilitatem, et glebe eius: vie vite ipsius aurum, propter multiplicem sanctitatem.

[Prima pars: Aurum = merita Iacobi ante mortem]. [Subdivision, les propriétés naturelles de l'or :] aurum in igne non consumitur, in opere non frangitur, in precio non excellitur, in cibo a lepra preservat. [Subdivision, les mérites correspondants de Jacques:] Sic Iacobus habuit: strenuitatem et pacienciam in passione flagellorum, humilitatem et obedienciam in execucione preceptorum, caritatis excellenciam in dileccione proximorum, sanitatis efficaciam in predicacione verborum. Per predicacionem ad graciam revocavit, per dileccionem in gracia 
conservavit, per execucionem in gracia ampliavit, per passionem in gracia confirmavit. [Dilatatio :] Iob trigessimo primo (Job 31.24, 27, 30) : Putavi aurum robur meum et obrizo dixi fiducia mea ; letatus sum super diviciis meis multis et plurima reperit manus mea. Vidi solem cum fulgeret, et letatum est in abscondito cor meum. Non dedi ad peccandum guttur meum. [Glose de la dilatatio:] Robur : strenuitas ad persecucionem, Fiducia: humilitas ad execucionem, Letatur super diviciis: merita per operacionem, Sol: Christum aspiciebat per contemplacionem, Letatur in abscondito: ad rectam intencionem, Non dat ad peccandum guttur : ad salvandum per predicacionem.

[Secunda pars : Saphirus = miracula Iacobi post mortem]. [Subdivision, les propriétés naturelles $\mathbf{d u}$ saphir :] virtutem sanativam contra febres, preservativam contra venena, purgativam contra obscuracionem, letificativam contra melancoliam. [Subdivision, les mérites correspondants de Jacques :] Sic Iacobus restaurat dampna, preservat pericula, purgat peccata, confert dona. [ Dilatatio :] Ysaias quinquagessimo quarto (Is. 54.11-14) : Fundabo te in saphiris, ponam iaspides propugnacula tua et portas tuas in lapides sculptos, et omnes terminos tuos lapides desiderabiles, universos filios tuos doctos a Domino et multitudinem pacis filiis tuis.Et in iustitia fundaberis. [Glose de la dilatatio:] Fundatus in saphiris: resarcit dampna, iaspides propugnacula tua: deffendit pericula, lapides sculptos: reprimit desideria, lapides desiderabiles: diluit flagicia [dont exemplum : de illo qui quoddam peccatum commiserat valde grave... episcopus ad Iacobum misit...], universos filios tuos doctos a Domino : revelat abscondita, et multitudinem pacis filiis tuis : confert dona, in iustitia fundaberis : multiplicat merita. (IOHANNES AEGIDII ZAMORENSIS, HN : 1530-1534)

31 Juan Gil utilise des images similaires pour illustrer ses propres sermons. On retrouve ainsi dans son Breviloquium de vitiis et virtutibus ${ }^{55}$ l'évocation - courante par ailleurs - de l'or purifié par le feu :

Prima igitur ratio propter quam precipit et consulit almifluus Dei Filius, Ihesus Christus, ut patientiam habeamus est quia ipsa est anime purgatiua et mundifficatiua. Sicut enim aurum et argentum homines in fornace probantur et depurantur, ita homines a culpa in tribulatione purgantur. (...) Quia sicut ignis metalla clarificat scoriam tollendo et fecem consumendo, metalla etiam mollificat, ita etiam tribulatio clarificat et aperit pacientis hominis intelletum ad Deum cognoscendum et mollificat patientis hominis uoluntatem siue affectum ad Deum diligendum (IOHANNES AEGIDII ZAMORENSIS, Breviloqvivm, éd. ATANASIO, 2015 : p. 143)

Le verset thématique utilisé par Juan Gil dans son De auro: Job 28.6 (Locus saphiri lapides eius et glebe illius aurum), se retrouve dans la troisième distinctio de la notice sur le saphir de Marc :

[Classification] Tertio, [Auctoritas scientifique] ut dicunt iidem [i.e., Isidorus, Dioscorides]. [Proprietas] Habet stellam in se refulgentem. [Similitudo] Significat beatam Virginem quae Filium Dei iustitiae solem et mundi lucem in uentre portauit et se portasse iugiter repraesentat. [Auctoritas biblique 1] Eccli. 24 : Qui creauit me, singulare scilicet templum sibi, requieuit in tabernaculo meo (Sir. 24.12), id est in utero clauso uirgineo suo benedicto reclinatorio et Spiritus sancti sempiterno sacrario. [Auctoritas biblique 2] Iob 28 : Locus sapphiri lapides eius, glebae illius aurum (Job 28.6). (MARCUS URBEVETENSIS, LM 7.82)

Là où Juan Gil utilise le lemme thématique Locus saphiri eius, glebe illius aurum pour moraliser l'or, l'assimilant à saint Jacques, Marc y a donc recours pour moraliser le saphir, qu'il assimile à la Vierge. Là où Gil n'emploie qu'un objet de comparaison - saint Jacques -, Marc en propose quinze, sans faire mention de saint Jacques. Seule l'autorité biblique coïncide. Il n'y a donc eu, semble-t-il, ni collaboration de deux auteurs, ni même influence. Les outils sont en revanche similaires. 


\section{La Vierge, l'or et le saphir : un exemple de sources similaires}

Rappelons que seule la lettre A est conservée pour l'HN de Gil. Les comparaisons entre Marc et Juan Gil ne sont donc possibles que pour les éléments dont les noms commencent par cette lettre A. Mais elles ne peuvent avoir lieu : on ne trouve aucune coïncidence dans les moralisations mariales. Marie est évoquée dans dix-huit moralisations de Marc d'Orvieto: De luna, De polo artico, De terra, De aquila, De ostrea, De cedro, De floribus, De flore campi, De germine, De palma, De pino, De rosa, De storace, De uirga, De uiola, De auro, De gelacia, De sapphiro. Elle l'est quatre fois chez Juan Gil de Zamora : De aere, De alumine, De aqua, De aurora. La comparaison par contre peut se faire pour le De auro. Mais elle tourne court : les deux frères mineurs n'usent pas de la même similitudo pour traiter du même objet: saint Jacques pour Juan Gil et la Vierge pour Marc.

En revanche, il est intéressant de noter les échos rencontrés par le De auro et le De sapphiro de Marc dans les sermones uirginales inédits de Juan Gil. Les deux franciscains ont probablement bu à des sources semblables, même s'il est vrai que, pour la Vierge, les références utilisées sont attendues: livres bibliques sapientiaux, recueils de miracles mariaux, Bernard de Clairvaux, le De Assumptione du pseudo-Jérôme Paschase Radbert, ou encore les Postilles de Hugues de Saint-Cher par exemple. Les coïncidences n'étant pas littérales, elles ne témoignent pas d'une collaboration ou d'une consultation des mêmes ouvrages mais d'une culture et d'une démarche communes. Fort diverses, ces coïncidences illustrent la similarité de formation et traduisent l'usage d'outils à la portée d'un compilateur franciscain de la fin du XIII ${ }^{e}$ siècle :

\begin{tabular}{|c|c|c|c|c|}
\hline $\begin{array}{l}\text { MARCUS } \\
\text { URBEVETENSIS }\end{array}$ & Texte extrait du LM & $\begin{array}{l}\text { IOHANNES AEGIDII } \\
\text { ZAMORENSIS }\end{array}$ & Textes égidiens & Source utilisée \\
\hline $\begin{array}{l}\text { De auro, } \\
7.15 .1\end{array}$ & 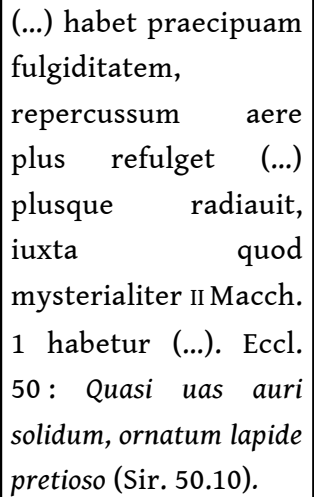 & 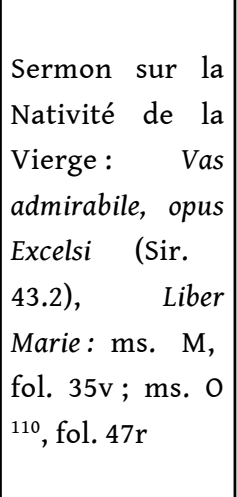 & $\begin{array}{l}\text { Facie pulcherrima, } \\
\text { spiritu sanctissima et in } \\
\text { utroque prefulgida super } \\
\text { omne uas aureum et } \\
\text { omnem r lapidem } \\
\text { preciosum, de } \text { quo } \\
\text { Ecclesiastici } \text { L dicitur } \\
\text { quod fuit quasi uas auri } \\
\text { solidum ornatum omni } \\
\text { lapide precioso (Sir. 50.10). }\end{array}$ & Sir. 50.10 \\
\hline
\end{tabular}




\begin{tabular}{|c|c|c|c|c|}
\hline $\begin{array}{l}\text { De auro, } \\
7.15 .1\end{array}$ & $\begin{array}{l}\text { Habetur etiam quod } \\
\text { post Ascensionem } \\
\text { Filii ad caelos, beatus } \\
\text { Dionysius et beatus } \\
\text { Ignatius prae claritate } \\
\text { radiante de facie eius } \\
\text { uix potuerunt semel } \\
\text { ferre aspectum } \\
\text { eiusdem. }\end{array}$ & $\begin{array}{l}\text { Sermon sur la } \\
\text { Nativité de la } \\
\text { Vierge: Aue } \\
\text { Maria gratia } \\
\text { plena (Luc. 1.28), } \\
\text { Opus sermonum : } \\
\text { ms. A, fol. } 84 \mathrm{va}\end{array}$ & $\begin{array}{l}\text { Vnde de ea legitur quod } \\
\text { cum uellet uidere eam } \\
\text { beatus Dionisius, rogauit } \\
\text { beatum Iohanem qui eam } \\
\text { custodiebat. At ille, } \\
\text { optenta licentia, antro } \\
\text { duxit beatum Dionisium } \\
\text { ubi orabat Virgo Maria. } \\
\text { Et in ipso introitu tantum } \\
\text { lumen uidit, et circa } \\
\text { ipsam, ut solis excederet } \\
\text { claritatem. Et stupefactus } \\
\text { ad uisionem, nec ualens } \\
\text { sustinere splendorem, } \\
\text { retrocessit et redux ad } \\
\text { beatum Iohanem } \\
\text { subplicans et exortans ne } \\
\text { illuc, quamquam } \\
\text { permitteret, introire ne } \\
\text { forte a simplicibus } \\
\text { putaretur esse Deus. }\end{array}$ & $\begin{array}{l}\text { Exemplum du } \\
\text { bienheureux } \\
\text { Denis, } \\
\text { identifié }\end{array}$ \\
\hline $\begin{array}{l}\text { De auro, } \\
7.15 .3\end{array}$ & $\begin{array}{l}\text { Significat beatae } \\
\text { Virginis } \\
\text { pulchritudinem et } \\
\text { elegantiam (...). Vnde } \\
\text { ad eam fertur ille } \\
\text { sermo, Cant. 4: Tota } \\
\text { pulchra es amica mea et } \\
\text { macula non est in te } \\
\text { (Cant. 4.7). }\end{array}$ & $\begin{array}{l}\text { Sermon sur la } \\
\text { Nativité de la } \\
\text { Vierge: Vas } \\
\text { admirabile, opus } \\
\text { Excelsi (Sir. } \\
43.2), \quad \text { Liber } \\
\text { Marie: ms. M, } \\
\text { fol. } 35 \mathrm{v} ; \mathrm{ms} . \mathrm{O} \\
110, \text { fol. } 47 \mathrm{r}\end{array}$ & $\begin{array}{l}\text { Tota pulcra est et macula } \\
\text { non est in ea (Cant. 4.7). } \\
\text { Facie pulcherrima, } \\
\text { spiritu sanctissima et in } \\
\text { utroque prefulgida super } \\
\begin{array}{l}\text { omne uas aureum et } \\
\text { omnem } \\
\text { preciosum }\end{array}\end{array}$ & Cant. 4.7 \\
\hline $\begin{array}{l}\text { De auro, } \\
7.15 .4\end{array}$ & $\begin{array}{l}\text { Vnde in igne non } \\
\text { consumitur, } r \text { sed } \\
\text { solum si quid habet } \\
\text { scoriae } \\
\text { impuritatis annexum, } \\
\text { ui caloris deponitur; } \\
\text { propter quod amplius } \\
\text { depuratur et clarius } \\
\text { siue fulgentius } \\
\text { inuenitur. Significat } \\
\text { in beata Virgine (...) } \\
\text { constantiam (...), } \\
\text { iuxta illud Eccli. 27: } \\
\text { Vasa figuli probat } \\
\text { fornax (Sir. 27.6). }\end{array}$ & $\begin{array}{l}\text { Sermon sur la } \\
\text { Nativité de la } \\
\text { Vierge: Vas } \\
\text { admirabile, opus } \\
\text { Excelsi (Sir. } \\
\text { 43.2), Liber } \\
\text { Marie: ms. M, } \\
\text { fol. } 35 \mathrm{v} ; \mathrm{ms} . \mathrm{O} \\
110, \text { fol. } 47 \mathrm{r}\end{array}$ & $\begin{array}{l}\text { Primo igitur Maria Virgo } \\
\text { melliflua fuit uas } \\
\text { mirabiliter preciosum } \\
\text { propter ipsius naturam, } \\
\text { seu materiam pretiosam, } \\
\text { que a scoria seu rubigine } \\
\text { originalis peccati } \\
\text { depurata et sanctificata, } \\
\text { egressa fuit hodie de } \\
\text { materno utero, sicut } \\
\text { egreditur uas preciosum } \\
\text { depuratum a scoria } \\
\text { materiali de clibano seu } \\
\text { fornace metallum, } \\
\text { quidem quanto purius } \\
\text { tanto preciosius. }\end{array}$ & \\
\hline
\end{tabular}




\begin{tabular}{|c|c|c|c|c|}
\hline $\begin{array}{l}\text { De auro, } \\
7.15 .5\end{array}$ & $\begin{array}{l}\text { Sic beata Virgo inter } \\
\text { omnia angelorum } \\
\text { agmina et hominum } \\
\text { est maioris uirtutis et } \\
\text { dignitatis (...). Prou. } \\
\text { ultimo: Multae filiae } \\
\text { congregauerunt } \\
\text { diuitias, tu supergressa } \\
\text { es uniuersas (Prou. } \\
\text { 31.29). }\end{array}$ & $\begin{array}{l}\text { Sermon sur la } \\
\text { Nativité de la } \\
\text { Vierge: Aue } \\
\text { gratia plena } \\
\text { (Luc. 1.28), Opus } \\
\text { sermonum: ms. } \\
\text { A, fol. 79ra; } \\
\text { Liber Marie: ms. } \\
\text { M, fol. 25v et } \\
\text { ms. } \mathrm{O}^{110}, \text { fol. } 35 \mathrm{v}\end{array}$ & $\begin{array}{l}\text { In quo tunc que sola } \\
\text { solem iustitie genuit, non } \\
\text { humanas tantum sed } \\
\text { angelicas etiam stellas } \\
\text { supergressa } \\
\text { uniuersas. }\end{array}$ & Prou. 31.29 \\
\hline $\begin{array}{l}\text { De auro, } \\
7.15 .6\end{array}$ & 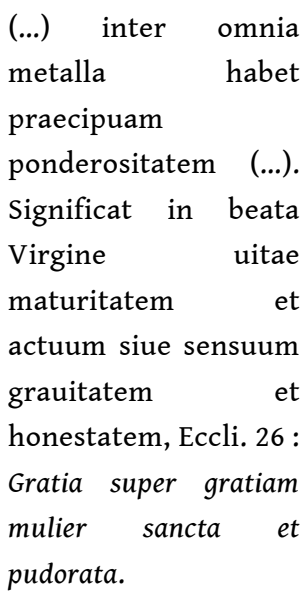 & $\begin{array}{l}\text { Sermon sur la la } \\
\text { Nativité de } \\
\text { Vierge: Aue } \\
\text { gratia plena } \\
\text { (Luc. 1.28), Opus } \\
\text { sermonum: ms. } \\
\text { A, fol. } 79 \mathrm{ra} \text {; } \\
\text { Liber Marie: ms. } \\
\text { M, fol. 25v et } \\
\text { ms. } \mathrm{O}^{110}, \text { fol. } 35 \mathrm{v}\end{array}$ & $\begin{array}{l}\text { Ipsa enim Virgo Maria } \\
\text { fuit sanctificata in } \\
\text { mente, fuit pudorata in } \\
\text { carne, } \\
\text { incomparabilis } \\
\text { imponderabilis uel } \\
\text { utroque. Et in signum } \\
\text { hoc dicitur Ecclesiastico } \\
\text { xxvi f: Gratia super } \\
\text { gratiam mulier sancta et } \\
\text { pudorata. }\end{array}$ & Sir. 26.19-20 \\
\hline $\begin{array}{l}\text { De auro, } \\
7.15 .7\end{array}$ & $\begin{array}{l}\text { Bernardus Super } \\
\text { 'Missus est' et alibi : } \\
\text { "In dubiis, in } \\
\text { periculis, in } \\
\text { necessitatibus, inuoca } \\
\text { Mariam; ipsa enim } \\
\text { est » etc. }\end{array}$ & $\begin{array}{l}\text { Sermon sur la la } \\
\text { Nativité de } \\
\text { Vierge: Aue } \\
\text { gratia plena } \\
\text { (Luc. 1.28), Opus } \\
\text { sermonum: ms. } \\
\text { A, fol. 79va; } \\
\text { Liber Marie: ms. } \\
\text { M, fol. 26r et } \\
\text { ms. } \mathrm{O}^{110}, \text { fol. } 36 \mathrm{v}\end{array}$ & $\begin{array}{l}\text { Huic etiam beatus } \\
\text { Bernardus in omeliis } \\
\text { Super missus est : si } \\
\text { «inter procellas huius } \\
\text { seculirideris } \\
\text { fluctuare, deuider } \\
\text { respice, uoca Mariam. In } \\
\text { periculis, in angustiis, in } \\
\text { rebus dubiis, Mariam } \\
\text { cogita, Mariam inuoca. » }\end{array}$ & $\begin{array}{l}\text { BERNARDUS } \\
\text { CLARAEVALLENSIS, ̀̀ } \\
\text { la louange de la } \\
\text { Vierge Mère: } \\
\text { 168-170 }\end{array}$ \\
\hline
\end{tabular}




\begin{tabular}{|c|c|c|c|c|}
\hline $\begin{array}{l}\text { De sapphiro, } \\
7.82 .1\end{array}$ & $\begin{array}{l}(\text {...) sola inter } \\
\text { mulieres gratia plena } \\
\text { et benedicta, Luc. 1, } \\
\text { describitur. } \\
\text { Secundum } \\
\text { Hieronymum : } \\
\text { «Gratia ceteris per } \\
\text { partes praestatur, } \\
\text { Mariae uero se totam } \\
\text { infudit [sic] gratiae } \\
\text { plenitudo et } \\
\text { diuinitatis unda ». }\end{array}$ & 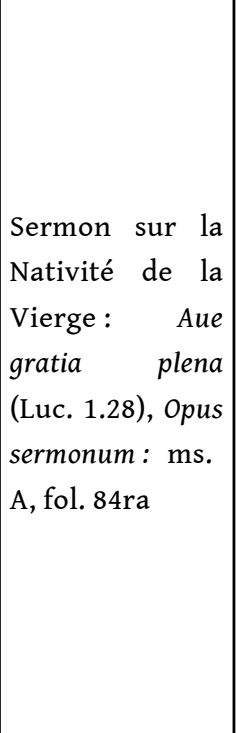 & $\begin{array}{l}\text { Et merito, quia } \\
\text { secundum positionem de } \\
\text { Assumptione eius : } \\
\text { «Sicut in comparatione } \\
\text { Dei, nemo bonus, ita et in } \\
\text { comparatione Matris } \\
\text { Domini nulla perfecta } \\
\text { inuenitur, } \\
\text { quantumcumque eximia } \\
\text { comprobetur. Et ideo, } \\
\text { inquit, bene plena quia } \\
\text { ceteris per partes } \\
\text { prestatur, Marie uero } \\
\text { simul se totam infundit } \\
\text { plenitudo gratie " (...): } \\
\text { Aue igitur gratia plena }\end{array}$ & $\begin{array}{l}\text { PASCHASIUS } \\
\text { RADBERTUS, De } \\
\text { Assumptione } \\
\text { sanctae Mariae } \\
\text { uirginis: } 106\end{array}$ \\
\hline $\begin{array}{l}\text { De sapphiro, } \\
7.82 .2\end{array}$ & $\begin{array}{l}\text { In colore caelo sereno } \\
\text { est simillimus. } \\
\text { Significat r beatam } \\
\text { Virginem } \\
\text { animam cum corpore } \\
\text { habuit semper totam } \\
\text { serenam et caelicam, } \\
\text { absque nube peccati }\end{array}$ & 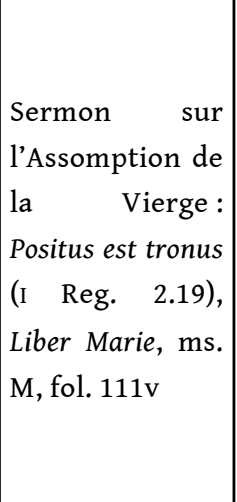 & $\begin{array}{l}\text { Qualis autem sit tronus } \\
\text { prehibitus, manifestat } \\
\text { sermo profeticus cum } \\
\text { subiungit } 15 \text { quod } \\
\text { similitudo troni erat } \\
\text { sicut aspectus lapidis } \\
\text { saphiri. Saphyrus, ut } \\
\text { dicitur in Glossa super } \\
\text { Apocalipsim : "Similis } \\
\text { est sereno celo ", habens } \\
\text { colorem celestem. }\end{array}$ & $\begin{array}{l}\text { HUGO DE SANCTO } \\
\text { CARO, Postilla, Liber } \\
\text { Apocalypsis : } 426\end{array}$ \\
\hline $\begin{array}{l}\text { De sapphiro, } \\
7.82 .3\end{array}$ & $\begin{array}{l}\text { Iob } 28 \text { : Locus sapphiri } \\
\text { lapides eius, glebae } \\
\text { illius aurum. }\end{array}$ & $\begin{array}{l}\text { Historia naturalis } \\
\text { De auro : } 1530\end{array}$ & $\begin{array}{l}\text { et idcirco in ipsius } \\
\text { preconium potest summi } \\
\text { verbum illud quod } \\
\text { scribitur Iob vigessimo } \\
\text { octavo: Locus saphiri } \\
\text { lapides eius et glebe illius } \\
\text { aurum. }\end{array}$ & Job 28.6 \\
\hline $\begin{array}{l}\text { De sapphiro, } \\
7.82 .4\end{array}$ & $\begin{array}{l}\text { habet uim ueneno } \\
\text { contrariam et ipsius } \\
\text { penitus exstinctiuam. }\end{array}$ & $\begin{array}{l}\text { Sermon sur la } \\
\text { Conception de } \\
\text { la } \quad \text { Vierge : } \\
\text { Dominus in celo } \\
\text { (Ps. 102.19), } \\
\text { Liber Marie, ms. } \\
\mathrm{M}, \text { fol. } 54 \mathrm{v} ; \mathrm{ms} . \\
\mathrm{O}^{110}, \text { fol. } 74 \mathrm{r}\end{array}$ & $\begin{array}{l}\text { Celum est almiflua Virgo } \\
\text { Maria, sed ros est eius } \\
\text { misericordia. Nam sicut } \\
\text { ros materialis estum } \\
\text { refrigerat, uenenum, } \\
\text { secundum sapientes, in } \\
\text { serpentibus reprimit et } \\
\text { coartat, et nichilominus } \\
\text { terram fecundat, ita } \\
\text { Virgo Maria refrigerat } \\
\text { estum temporalium } \\
\text { tribulationum, reprimit } \\
\text { uenenum criminalium } \\
\text { suggestionum }\end{array}$ & \\
\hline
\end{tabular}




\begin{tabular}{|c|c|c|c|}
\hline $\begin{array}{l}\text { De sapphiro, } \\
7.82 .6\end{array}$ & $\begin{array}{l}\text { lar } \\
\\
\text { Exemplum etiam in } \\
\text { multis } \\
\text { materialiter educit } \\
\text { tota die miraculos de } \\
\text { captiuitatibus, sicut } \\
\text { habetur in suis } \\
\text { miraculis. }\end{array}$ & 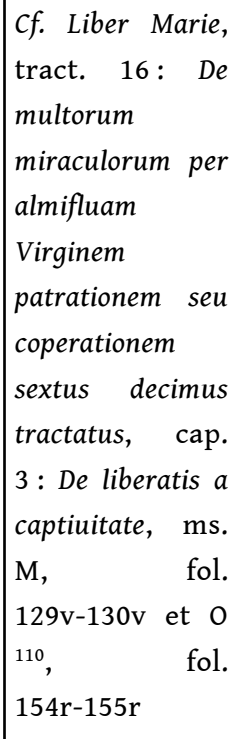 & \\
\hline $\begin{array}{l}\text { De sapphiro, } \\
7.82 .9\end{array}$ & \begin{tabular}{|lr} 
& \\
& \\
merita et & orationes \\
beatae & Mariae \\
remittunt & et \\
refrigerant rebres & febre \\
concupiscentiae, irae \\
et superbiae & et \\
huiusmodi &
\end{tabular} & $\begin{array}{l}\text { Idée courante } \\
\text { dans l'Opus } \\
\text { sermonum : } \\
\text { sermons sur la } \\
\text { Vierge Lux orta } \\
\text { est iusto (Ps. } \\
96.11), \quad \text { Aue } \\
\text { gratia rlena } \\
\text { (Luc. 1.28), } \\
\text { Dominus in celo } \\
\text { (Ps. 102.19), et } \\
\text { dans le Liber } \\
\text { Marie: } \text { Meditatio } \\
\text { duodecima beate } \\
\text { Virginis incipiens } \\
\text { a M littera }\end{array}$ & \\
\hline
\end{tabular}

\section{Job et l'argent : une liste de mots-clefs}

Un autre parallèle illustre cette fois une similarité non plus de sources mais de procédés. Juan Gil comme Marc établissent dans le cours de leur développement une liste de motsclefs, propriétés de la matière traitée, qui sont autant de parties possibles d'un sermon. La démarche est visible dans la rubrique De argento de l'un comme de l'autre. Huit caractéristiques attribuées à Isidore sont énumérées par Marc : principium, album, clarum, sonorum, purum, pretiosum, conseruatiuum, curatiuum. On retrouve les mêmes chez Juan Gil, qui finit par en sélectionner une partie, utilisée pour construire son sermon.

Chez Marc d'Orvieto, une octuple distinctio découle de cette liste. En voici la structure et les sources (MARCUS URBEVETENSIS, LM, 6.16 : 1213-1216) ${ }^{56}$ :

Argentum habet has condiciones siue proprietates. 


\begin{tabular}{|c|c|}
\hline \multicolumn{2}{|l|}{ Primo } \\
\hline $\begin{array}{l}\text { Caractéristique et } \\
\text { autorité scientifique } \\
\text { Autorité implicite : BARTHOLOMAEUS } \\
\text { ANGLICUS, PR, lib. XVI De lapidibus, cap. VIII } \\
\text { De argento uiuo, Frankfurt, } 1601, \text { p. } 721^{57}\end{array}$ & $\begin{array}{l}\text { Primo enim ut dicitur in Plateario, est omnium } \\
\text { metallorum principium. }\end{array}$ \\
\hline Moralisation & $\begin{array}{l}\text { Significat scripturam uenerabilem esse caput et } \\
\text { principium omnis philosophicae et rhetoricae } \\
\text { eloquentiae siue elegantiae. }\end{array}$ \\
\hline $\begin{array}{l}\text { Autorité vétéro-testamentaire } \\
\text { Job } 28.1\end{array}$ & $\begin{array}{l}\text { Et hoc innuitur Iob } 28 \text { : Habet argentum uenarum } \\
\text { suarum principia etc. }\end{array}$ \\
\hline $\begin{array}{l}\text { Autorité antique } \\
\text { CASSIODORUS, Super Psalmos, MIGNE éd., } \\
\text { praef. : } 15\end{array}$ & $\begin{array}{l}\text { Vnde dicitur Cassiodorus Super Psalterium quod omnis } \\
\text { poeticae et rhetoricae eloquentiae ornatus et } \\
\text { elegantia a sacra Scriptura sumpsit initium. }\end{array}$ \\
\hline $\begin{array}{l}\text { Autorité patristique } \\
\text { AUGUSTINUS HIPPONENSIS, De civitate Dei, } \\
\text { MIGNE éd., } 18.39\end{array}$ & $\begin{array}{l}\text { Augustinus etiam dicit De ciuitate libro XVIII : Nullos } \\
\text { ante patriarchas nostros potuisse se iactare, id est de } \\
\text { antiquitate sapientiae et de huiusmodi. }\end{array}$ \\
\hline \multicolumn{2}{|l|}{ Secundo } \\
\hline $\begin{array}{l}\text { Caractéristique et } \\
\text { autorité scientifique } \\
\text { Cf. ISIDORUS HISPALENSIS, Etymologiae, MIGNE } \\
\text { éd., } 16.19 .1\end{array}$ & $\begin{array}{l}\text { Secundo, ut dicit Isidorus, argentum est album, quod } \\
\text { patet ex sui aspectu. }\end{array}$ \\
\hline Moralisation & Significat angelicam castitatem \\
\hline $\begin{array}{l}\text { Autorité néo-testamentaire } \\
\text { Apoc. } 3.4\end{array}$ & $\begin{array}{l}\text { quam praedicat uenerabilis Scriptura, Apoc. } 3: \text { Habes } \\
\text { pauca nomina in Sardis, qui non inquinauerunt uestimenta } \\
\text { sua; ambulabunt mecum in albis quia digni sunt. }\end{array}$ \\
\hline $\begin{array}{l}\text { Autorité néo-testamentaire } \\
\text { Apoc. } 3.18\end{array}$ & Et infra: ut uestimentis albis induaris etc. \\
\hline $\begin{array}{l}\text { Autorité hagiographique } \\
\text { Vita S. Caeciliae : MOMBRITIUS, 334.20-24 }\end{array}$ & $\begin{array}{l}\text { Vnde, quia beata Cecilia et Valerianus imitati sunt } \\
\text { angelicam castitatem, allatae sunt eis duae coronae de } \\
\text { caelo liliis albescentes et niueo candore uernantes. }\end{array}$ \\
\hline \multicolumn{2}{|l|}{ Tertio } \\
\hline $\begin{array}{l}\text { Caractéristique et } \\
\text { autorité scientifique } \\
\text { ISIDORUS HISPALENSIS }\end{array}$ & Tertio, ut dicit idem, argentum est clarum. \\
\hline Moralisation & Significat claritatem Scripturae uenerabilis, \\
\hline
\end{tabular}




\begin{tabular}{|c|c|}
\hline $\begin{array}{l}\text { Autorité vétéro-testamentaire } \\
\text { Sap. } 6.13\end{array}$ & Sap. 6 : Clara est et quae numquam marcescit sapientia. \\
\hline $\begin{array}{l}\text { Autorité vétéro-testamentaire } \\
\text { Sap. } 7.25\end{array}$ & $\begin{array}{l}\text { Item, } 7: \text { Emanatio quaedam claritatis omnipotentis Dei } \\
\text { sincera, et ideo nihil inquinatum in illam incurrit. }\end{array}$ \\
\hline $\begin{array}{l}\text { Autorité patristique } \\
\text { AUGUSTINUS HIPPONENSIS, Confessiones } 6.2 .2\end{array}$ & $\begin{array}{l}\text { Vnde Augustinus in libro Confessionum uocat } \\
\text { Ambrosium doctorem huius praeclarae } \\
\text { praedicatorem ueritatis et antistitem pietatis. }\end{array}$ \\
\hline \multicolumn{2}{|l|}{ Quarto } \\
\hline $\begin{array}{l}\text { Caractéristique et } \\
\text { autorité scientifique } \\
\text { ISIDORUS HISPALENSIS }\end{array}$ & Quarto, ut dicit idem, argentum est sonorum. \\
\hline Moralisation & $\begin{array}{l}\text { Sic sancta Scriptura est eloquentissima et } \\
\text { uniuersaliter praedicata. }\end{array}$ \\
\hline $\begin{array}{l}\text { Autorité vétéro-testamentaire } \\
\text { Ps. } 18.5\end{array}$ & Vnde in Ps. 18 : In omnem terram exiuit sonus eorum etc. \\
\hline $\begin{array}{l}\text { Autorité néo-testamentaire } \\
\text { Col. } 1.23\end{array}$ & $\begin{array}{l}\text { Item, Col. } 1 \text { : Euangelium praedicatum est in uniuersa } \\
\text { creatura. }\end{array}$ \\
\hline $\begin{array}{l}\text { Autorité théologique } \\
\text { Cf. ANSELMUS CANTUARIENSIS, Epistolae, } \\
\text { sCMITT éd., } 313\end{array}$ & $\begin{array}{l}\text { In huius argumentum apostoli dispersi sunt per totum } \\
\text { mundum, et non est qui se excusare possit a lingua } \\
\text { ueritatis, secundum Anselmum, Cur Deus homo. }\end{array}$ \\
\hline \multicolumn{2}{|l|}{ Quinto } \\
\hline $\begin{array}{l}\text { Caractéristique et } \\
\text { autorité scientifique } \\
\text { ISIDORUS HISPALENSIS }\end{array}$ & Quinto, ut dicit idem, argentum est purum. \\
\hline Moralisation & $\begin{array}{l}\text { Sic sancta Scriptura nihil continet falsum uel } \\
\text { absurdum. }\end{array}$ \\
\hline $\begin{array}{l}\text { Autorité vétéro-testamentaire } \\
\text { Ps. } 11.7\end{array}$ & $\begin{array}{l}\text { Ps. 11: Eloquia Domini, eloquia casta, argentum igne } \\
\text { examinatum, probatum terrae, purgatum septuplum. }\end{array}$ \\
\hline $\begin{array}{l}\text { Autorité patristique } \\
\text { AUGUSTINUS HIPPONENSIS, Contra Faustum, } \\
\text { MIGNE éd., } 11.5\end{array}$ & $\begin{array}{l}\text { Vnde dicit Augustinus Contra Faustum xI libro: «Noli } \\
\text { dicere quod Scriptura aliquid contineat erroneum uel } \\
\text { absurdum, sed si ibi aliquid tale inueneris, unum } \\
\text { horum trium tibi elige, scilicet quod uel interpes } \\
\text { errauit, uel codex est mendosus, uel tu non } \\
\text { intelligis». }\end{array}$ \\
\hline & \\
\hline
\end{tabular}




\begin{tabular}{|c|c|}
\hline $\begin{array}{l}\text { Caractéristique et } \\
\text { autorité scientifique } \\
\text { ISIDORUS HISPALENSIS }\end{array}$ & Sexto, ut dicit idem, argentum est pretiosum. \\
\hline Moralisation & Significat Scripturam uenerabilem \\
\hline $\begin{array}{l}\text { Autorité vétéro-testamentaire } \\
\text { Prou. } 3.15\end{array}$ & $\begin{array}{l}\text { quae, secundum illud Prou. } 3 \text { : Pretiosior est cunctis } \\
\text { opibus etc. }\end{array}$ \\
\hline $\begin{array}{l}\text { Autorité vétéro-testamentaire } \\
\text { Sap. } 7.9\end{array}$ & Et Sap. 7 : Nec comparaui illi lapidem pretiosum etc. \\
\hline $\begin{array}{l}\text { Autorités patristiques } \\
\text { IOANNES CHRYSOSTOMUS, Super Genesim, } \\
\text { MIGNE éd., } 21.1\end{array}$ & $\begin{array}{l}\text { Vnde dicit Chrysostomus et Damascenus similiter in } \\
\text { diuersis tractatibus quod maius omnium donorum a } \\
\text { Deo homini concessorum et pretiosius est sancta } \\
\text { Scriptura. }\end{array}$ \\
\hline \multicolumn{2}{|l|}{ Septimo } \\
\hline $\begin{array}{l}\text { Caractéristique et } \\
\text { autorité scientifique } \\
\text { BARTHOLOMAEUS ANGLICUS PR } 16.8^{58}\end{array}$ & $\begin{array}{l}\text { Septimo, ut dicitur in Lapidario, argentum est balsami } \\
\text { uirtuositatis potissime conseruatiuum; similiter } \\
\text { uirtutis in iaspide iuuatiuum. Maiorem enim uirtutem } \\
\text { habet iaspis in argento quam in auro, ut dicitur } \\
\text { ibidem }^{59} \text {. }\end{array}$ \\
\hline Moralisation & $\begin{array}{l}\text { Significat uenerabilem Scripturam studentium in se et } \\
\text { adamantium se esse conseruatiuam penitus bonae } \\
\text { famae et iuuare potissime in uirtute uirentes fide et } \\
\text { amoenitate laetitiae aeternae uitae ad } \\
\text { repraesentationem iaspidis qui est colore uiridis et } \\
\text { uirtute singulari confert laetitiam et confortationem. }\end{array}$ \\
\hline $\begin{array}{l}\text { Autorité vétéro-testamentaire } \\
\text { Sap. } 8.10 .16\end{array}$ & $\begin{array}{l}\text { Vnde Sap. } 8: \text { Habebo propter hanc claritatem ad turbas et } \\
\text { honorem apud seniores. Intrans in domum meam } \\
\text { conquiescam cum illa; non enim habet amaritudinem } \\
\text { conuersatio illius, neque tedium conuictus illius, sed } \\
\text { laetitiam et gaudium. }\end{array}$ \\
\hline $\begin{array}{l}\text { Autorité patristique } \\
\text { AUGUSTINUS HIPPONENSIS, Conf. : 9.10.22-23 }\end{array}$ & $\begin{array}{l}\text { Et hoc est Augustinus dicit, IX Confessionum : «Accepta } \\
\text { baptismi gratia positus apud ostia Tiberina cum marte } \\
\text { mea, colloquebamur soli ualde dulciter. Et inhiabamus } \\
\text { ore cordis pariter in superna fluenta fontis uitae, } \\
\text { uilescebatque mundus iste » etc. }\end{array}$ \\
\hline Octauo & \\
\hline
\end{tabular}




\begin{tabular}{|c|c|}
\hline $\begin{array}{l}\text { Caractéristique et } \\
\text { autorité scientifique } \\
\text { BARTHOLOMAEUS ANGLICUS PR } 16.8^{60}\end{array}$ & $\begin{array}{l}\text { Octauo, ut dicit Platearius, inter metalla post aurum in } \\
\text { efficacia obtinet principatum. Est enim medicinale et } \\
\text { multarum aegritudinem curatiuum. Nam eius spuma } \\
\text { uulnera anat et replet, et in eis carnes superfluas } \\
\text { crescere non permittit. Cicatrices obducit, sordes } \\
\text { corporis mundificat, a foetore custodit, confortat et } \\
\text { restringit fluxibilia membra. }\end{array}$ \\
\hline Moralisation & $\begin{array}{l}\text { Significat uenerabilem Scripturam post aurum gloriae } \\
\text { et gratiae Dei in efficacia et utilitate salutis humanae } \\
\text { obtinere inter omnia alia dona principatum et efficere } \\
\text { in animabus spiritualiter et correspondenter } \\
\text { quaecumque facit aurum medicinaliter in corporibus } \\
\text { materialiter, ut tactum est. Sicut de plano adaptare } \\
\text { potest per singula Deo deuotus lector et Scripturae } \\
\text { ipsius cultoe. }\end{array}$ \\
\hline $\begin{array}{l}\text { Autorité vétéro-testamentaire } \\
\text { Prou. } 10.20\end{array}$ & $\begin{array}{l}\text { Vnde de ea dicitur, Prou. } 10 \text { : Argentum electum, lingua } \\
\text { iusti. }\end{array}$ \\
\hline $\begin{array}{l}\text { Autorité vétéro-testamentaire } \\
\text { Prou. } 8.19\end{array}$ & Et Prou. 8 : Meliora sunt genimina mea argento electo. \\
\hline $\begin{array}{l}\text { Autorité théologique } \\
\text { ANSELMUS CANTUARIENSIS, De concordia } \\
\text { praescentiae et praedestinationis nec non } \\
\text { gratiae Dei, sCHMITT éd., } 3.6\end{array}$ & $\begin{array}{l}\text { Vnde dicit Anselmus Cur Deus homo et alibi, quod nihil } \\
\text { utile possumus loqui uel scientiae quod in ea non sit. }\end{array}$ \\
\hline
\end{tabular}

Examinons à présent le traitement réservé à l'argent par Juan Gil: sa notice encyclopédique est suivie d'une double moralisation, en forme de sermon bipartite et dépourvu de doxologie. Ces deux parties traitent l'une de l'argent, l'autre de l'or, car le plan choisi par Juan Gil dépend de la divisio du thema choisi : Habet argentum venarum suarum principia et auro locus est in quo conflatur (Job 28.1) - ce lemme se retrouve d'ailleurs dans la première distinctio de Marc. La notice, les moralisations et le sermon de Juan Gil sont reproduits dans le tableau ci-dessous. J'en propose les sources à nouveaux frais, son éditeur ayant simplement déclaré Vincent de Beauvais comme source générale de la notice, ce qui ne se vérifie pas ici. Par ailleurs, l'éditeur n'ayant pas discerné que le développement de la moralisation est un sermon, il a fallu dans ce tableau procéder à un découpage et l'expliciter (IOHANNES AEGIDII ZAMORENSIS, HN, 1026-1030) :

\begin{tabular}{|l|l}
\hline Sources & Notice encyclopédique
\end{tabular}




\begin{tabular}{|c|c|}
\hline $\begin{array}{l}\text { Bartholomeus Anglicus PR } 16.7 \text { : «De } \\
\text { argento » } \\
\text { Dont autorités scientifiques } \\
\text { explicites: Ricardus Rufus (cf. } \\
\text { RUPERTUS TUITENSIS, Commentaria in } \\
\text { Canticum Canticorum, HAACKE éd., 5.581, } \\
\text { p. 120), Aristotiles Implicite : ISIDORUS } \\
\text { HISPALENSIS, Etymologiae: 16.19.1 } \\
\text { (Argentum apud - nigras reddat) }\end{array}$ & $\begin{array}{l}\text { Argentum apud Grecos «argirum " vocatur. Cui mirum } \\
\text { in modum accidit quod, cum sit candidum, impressum } \\
\text { corpori lineas nigras reddat. In eius composicione } \\
\text { concurrit argentum uiuum et sulphur album. Plus } \\
\text { autem est ibi de aureo et aqueo argenti uiui quam } \\
\text { sulphuris et ideo non est tanti ponderis sicut aurum, ut } \\
\text { dicit Ricardus Rufus. Compositum ergo argentum est } \\
\text { solidum compositum ex argento uiuo puro et sulphure } \\
\text { alio non urente, ut dicit Aristotiles. }\end{array}$ \\
\hline $\begin{array}{l}\text { BARTHOLOMEUS ANGLICUS PR 16.8: De } \\
\text { argento uiuo }\end{array}$ & $\begin{array}{l}\text { Est autem substancia eius alba ex claritate atque subtilis } \\
\text { et ex albedine terre bene subtilis et digesta. Habet eciam } \\
\text { et albedinem ex admixtione aeris cum predictis. }\end{array}$ \\
\hline $\begin{array}{l}\text { Cf. THOMAS CANTIMPRATENSIS, Liber de } \\
\text { natura rerum, BOESE, } 15.4: \text { De argento: } \\
376 \\
\text { Dont exemple biblique : Moïse }\end{array}$ & $\begin{array}{l}\text { Est autem hoc metallum, ut dictum est, album, clarum, } \\
\text { sonorum, purum, minus habens de ductibilitate quam } \\
\text { aurum. Bonum tinnitum habet et placidum, maxime si } \\
\text { eri misceatur. Ob hoc autem tubas argenteas ductiles } \\
\text { precepit Moyses fieri ad hostium terrorem et } \\
\text { exhortacionem ad bellum et ad movendum castra et ad } \\
\text { epulas convocandum. }\end{array}$ \\
\hline $\begin{array}{l}\text { BARTHOLOMEUS ANGLICUS PR } 16.8: \text { De } \\
\text { argento uiuo } \\
\text { Dont autorité scientifique explicite: } \\
\text { Lapidarius }\end{array}$ & $\begin{array}{l}\text { Balsami est conservatiuum et uirtutis in iaspide } \\
\text { iuvativum, maiorem enim virtutem habet iaspis in } \\
\text { argento quam in auro, ut dicitur in Lapidario. Liquabile } \\
\text { est in igne, resoluvitum et liquefactivum, colorem habet } \\
\text { igneum et rubeum; infrigidatum vero iterum efficitur } \\
\text { solidum atque durum. Et, quamvis sit grossum et } \\
\text { materiale respectu auri, tamen post aurum in valore et } \\
\text { precio inter metalla obtinet principatum. Et est } \\
\text { medicinale et multarum egritudinum curativum, nam } \\
\text { eius scoria sive spuma vulnera sanat et replet et carnes } \\
\text { superfluas in eis crescere non permittit; cicatricem } \\
\text { inducit, sordes corporis mundificat et abstergit, } \\
\text { cauterium a fetore custodit, confortat fluxibilia membra } \\
\text { et constringit. Ex contactu terre rubiginem contrahit, } \\
\text { sed ex arene et salis confricacione colorem pristinum } \\
\text { recipit et resumit. }\end{array}$ \\
\hline AVICENNA, Canon : 2.2.65 : Argentum & $\begin{array}{l}\text { Argentum solidum est, secundum Auicennam in } \\
\text { secundo libro Canonis : infrigidativum et exsiccativum ; } \\
\text { scoria enim est stiptica valde, et in ipsa est contraccio et } \\
\text { exsiccacio. Quando permiscetur confricacio eius } \\
\text { medicinis aliis, confert humiditatibus viscosis. Est valde } \\
\text { bonum scabiei et pruritui. Confricacio eius confert } \\
\text { fetori oris, cum permiscetur permixtionibus aliis. } \\
\text { Confricacio eius cum commixtionibus confert tremori } \\
\text { cordis. Hucusque Avicenna. }\end{array}$ \\
\hline
\end{tabular}


Argenti metallo comparari potest racionabiliter predicator, eo quod debet esse clarus, probatus vita, solidus in doctrina, sonorus fama ; uel probatus vita, solidus in doctrina, sonorus in lingua, eloquia enim Domini eloquitur casta argentum igne examinatum (Ps. 11.7).

\section{Moralisation spéciale}

Argento potest beati Pauli conversio et predicacio comparari.

Thema

Et ideo in eius commendacionem merito potest summi verbum illud quod scribitur Iobis vigessimo octavo: Habet argentum venarum suarum principia et auro locus est in quo conflatur (Job 28.1).

\section{Divisio}

Commendatur quidem ipsius predicacio sub methaphora argenti sonori, conversacio sub methaphora auri decori, conversio sub methaphora principii inchoantis, passio sub methaphora conflatorii expurgantis.

Prima pars

\begin{tabular}{|c|c|}
\hline Subdivision & $\begin{array}{l}\text { Circa primum notandum quod per argentum recte eius predicacio } \\
\text { designatur. Habet enim argentum uirtutem curatiuam, uirtutem } \\
\text { corrosivam, uirtutem purgatiuam, uirtutem confortativam. Nam argenti } \\
\text { scoria sive spuma sive limatura uulnera curat et carnibus superuestit, } \\
\text { carnes putridas destruit et corrodit, membra sordida mundificat et decorat, } \\
\text { membra fluida fortificat et vigorat. }\end{array}$ \\
\hline $\begin{array}{l}\text { Autorité néo- } \\
\text { testamentaire } \\
2 \text { Tim. } 4.2\end{array}$ & $\begin{array}{l}\text { Propterea dicebat Apostolus ad Thimotheum: Argue, scilicet delinquentes; } \\
\text { increpa diffidentes; obsecra penitentes in paciencia quantum ad pacientes, in } \\
\text { doctrina quantum ad proficientes ( } 2 \text { Tim. 4.2). Sic beatus Paulus in sua } \\
\text { predicacione curabat penitentes, corrodebat delinquentes, decorabat } \\
\text { proficientes, confortabat pacientes et proficientes. Et in hiis quatuor } \\
\text { consistit virtus predicacionis. Et hec omnia patent in suis epistolis. }\end{array}$ \\
\hline $\begin{array}{l}\text { Autorité vétéro- } \\
\text { testamentaire } \\
\text { Prou. } 10.20-22\end{array}$ & $\begin{array}{l}\text { Et propterea Sapiens, in spiritu prophetico predicacionis et doctrine beati } \\
\text { Pauli privilegium et excellenciam recognoscens, in commendacionem eius } \\
\text { prorumpit dicens : Lingua iusti argentum electum, cor autem impiorum perdicio. } \\
\text { Labia iusti erudiunt plurimos; qui autem indocti sunt in cordis egestate morientur. } \\
\text { Benediccio Domini divites facit, nec sociabitur eis affliccio (Prou. 10.20-22). Certe } \\
\text { lingua iusti, id est beati Pauli, fuit argentum electum et sonorum per } \\
\text { increpacionem delinquentum; erudit plurimos per instruccionem } \\
\text { penitentum; divites fecit per promocionem proficiencium; affliccionem } \\
\text { delevit per consolacionem pacientum. }\end{array}$ \\
\hline
\end{tabular}




\begin{tabular}{|c|c|}
\hline $\begin{array}{l}\text { Exemple } \\
\text { biblique } \\
\text { Paul }\end{array}$ & $\begin{array}{l}\text { Circa aurum notandum quod per aurum vita beati Pauli designatur propter } \\
\text { quatuor proprietates quibus aurum cetera metalla preminet et precellit. } \\
\text { Auro enim inter metalla nichil purius quoad materiam, nichil ductibilius } \\
\text { quoad operacionem. Sic beatus Paulus habuit puritatem quoad se ipsum, } \\
\text { caritatem quoad proximum, soliditatem pacientem quoad adversarium, } \\
\text { humilitatem obediencie quoad Deum. }\end{array}$ \\
\hline $\begin{array}{l}\text { Autorités néo- } \\
\text { testamentaires } \\
\text { Gal. } 5.24 ; \quad \text { Gal. } \\
6.14 ; 1 \text { Cor. } 13.2 ; \\
\text { Rom. } 8.18 ; 1 \text { Cor. } \\
15.9\end{array}$ & $\begin{array}{l}\text { Certe puritatem habebat qui dicebat: Qui Christi sunt carnem suam } \\
\text { crucifixerunt cum viciis et concupiscenciis (Gal. 5.24). Et iterum : Michi mundus } \\
\text { crucifixus est, et ego mundo (Gal. 6.14). Caritatem habebat qui dicebat: Si } \\
\text { habuero fidem ita ut montes transferam, caritatem autem non habeam, nichil sum } \\
\text { (1 Cor. 13.2). Pacienciam et propositum paciendi habebat qui dicebat: Non } \\
\text { sunt condigne passiones huius temporis ad futuram gloriam, que revelabitur in } \\
\text { nobis (Rom. 8.18). Humilitatem habebat qui dicebat: Ego sum minimus } \\
\text { apostolorum, qui non sum dignus, et cetera (1 Cor. 15.9). }\end{array}$ \\
\hline $\begin{array}{l}\text { Autorité vétéro- } \\
\text { testamentaire } \\
\text { Job } 22.23-25,28 \text {, } \\
29,30\end{array}$ & $\begin{array}{l}\text { Et propter hec omnia dicitur Iobis vigessimo secundo: Si reversus fueris ad } \\
\text { Deum, longe facies iniquitatem a tabernaculo tuo et dabit tibi pro terra silicem, et } \\
\text { pro silice torrentes aureos et ipse erit contra hostes tuos (Job 22.23-25). Certe, si } \\
\text { reversus fueris ad Dominum, scilicet per pacienciam, longe facies iniquitatem, } \\
\text { scilicet per mundiciam et innocenciam, et dabit tibi pro terra silicem, scilicet } \\
\text { pro malicia caritatem, sicut enim terra habet obscuritatem et } \\
\text { compaccionem, silex lapis emittit ignem per collissionem; pro silice torrentes } \\
\text { aureos, id est pro duricia humilitatem, sicut enim lapis habet duriciem et } \\
\text { resistenciam, torrens fluxum et defluenciam, aurum decorem et } \\
\text { resplendenciam; nam humilitas est virtutum omnium ornamentum. } \\
\text { Sequitur: Ipse erit contra hostes tuos, quia ipse infundet tibi ad sustinendum } \\
\text { virtutem et pacienciam. Et ista quatuor evidencius tangit auctoritas cum } \\
\text { subiungit: in viis tuis splendebit lumen (Job 22.28), quod pertinet ad } \\
\text { perfeccionem caritatis; qui humiliatus fuerit, erit in gloria (Job 22.29), et hoc } \\
\text { pertinet ad subieccionem humilitatis; qui inclinaverit oculos suos (Job 22.29), } \\
\text { scilicet ad sustinendum, ipse salvabitur, hoc pertinet ad virtutem paciencie; } \\
\text { salvabitur innocens in mundicia manuum suarum (Job 22.30), hoc pertinet ad } \\
\text { puritatem innocencie. }\end{array}$ \\
\hline
\end{tabular}

On le voit, dans le premier exemple, la moralisation de l'argent est la Sainte Écriture, dans le deuxième, la prédication, incarnée par saint Paul. Marc dévoile un arsenal complet de citations vétéro-testamentaires (Job, Proverbes, Sagesse, Psaumes) et néo-testamentaires (Apocalypse, Colossiens), patristiques (Augustin, Jean Chrysostome), théologiques (Anselme) voire hagiographiques (Vita Ceciliae). Juan Gil, s'il part du lemme de Job choisi par Marc dans son premier point, ne propose que des citations néo-testamentaires en cohérence avec son exemple, Paul: Lettres à Timothée, aux Colossiens, Galates, Corinthiens et Romains, avec deux « écarts » : un verset des Proverbes et le chapitre $22 \mathrm{de}$ Job qui sont toutefois appliqués aux vertus pauliniennes. Les sources diffèrent donc, la moralisation choisie par chacun est distincte.

En revanche, les mots-vedettes sont similaires. Marc a énuméré huit caractéristiques de la matière argent qui sont l'occasion pour lui de huit développements qui équivalent à huit parties possibles d'un sermon. Juan Gil, lui, énumère ces mêmes caractéristiques ( principium, album, clarum, sonorum, purum, pretiosum, conseruatiuum, curatiuum) dans la 
notice encyclopédique de l'argent. Mais lorsqu'il aborde la moralisation de l'argent et confectionne de toutes pièces un sermon pour illustrer cette moralisation, il ne sélectionne que certaines caractéristiques de l'argent (sonorus, curatiuus, purus) pour développer son propos.

\section{Conclusion}

41 Dans l'état actuel de la recherche, le franciscain Juan Gil de Zamora apparaît comme le seul encyclopédiste du XIII ${ }^{e}$ siècle à avoir poussé jusque dans ses ultimes conséquences l'usage homilétique des moralisations des res naturales. Les ouvrages moralisés, véritables collections prêtes-à-emporter pour le prédicateur, sont multiformes: similitudines, exempla, distinctiones..., et se nourrissent des informations données par les encyclopédies naturelles, y compris les encyclopédies moralisées comme le De proprietatibus rerum. Juan Gil y ajoute de véritables plans développés de sermons, utilisables en l'état, et assimilant une douzaine d'éléments naturels à une véritable galerie hagiographique. La causa scribendi d'une telle démarche, outre l'intention didactique et le souci d'édification, est vraisemblablement le désir de fournir un vademecum d'usage aisé pour le prédicateur.

L'encyclopédie la plus proche, dans le choix des procédés (mots-clefs, subdivisions), des sources et des objets (d'après un échantillon marial) demeure, à ce stade des travaux, le Liber de moralitatibus, attribué à Marc d'Orvieto, frère mineur contemporain. C'est en comparant la notice sur le sapin de ce Liber avec celle de l'Historia naturalis de Juan Gil qu'a été découverte une probable dépendance commune aux marginalia du De proprietatibus rerum de Barthélemy l'Anglais.

Les données encyclopédiques (notices naturelles et notes morales) réunies par la première génération de naturalistes enrichissent sans conteste l'art complexe de la prédication mendiante de la fin du siècle. Cet apport d'un genre à l'autre est-il direct ? Il y a eu fort probablement recours à de nouveaux outils (tables alphabétiques, dictionnaires d'éthique) et à l'amélioration d'outils plus anciens (recueils de propriétés du XII siècle). La parution d'éditions en cours (sermons de Juan Gil, encyclopédie de Barthélemy avec ses marginalia), l'élargissement de la comparaison aux œuvres des autres encyclopédistes qui nourrirent l'Historia naturalis de Juan Gil (Thomas de Cantimpré, Vincent de Beauvais) et l'examen d'outils de travail tels que les florilèges d'éthique, tables de matières et dictionnaires lexicographiques permettront certainement d'explorer plus avant le vaste champ de la matière prédicable et des pratiques de la prédication.

\section{BIBLIOGRAPHIE}

\section{Manuscrits utilisés}

Assise, Sacro convento, 414. 
Burgo de Osma, Archivo catedralicio, 110.

Madrid, Biblioteca Nacional de España, 9503.

Paris, Bibliothèque Nationale de France, lat. 16099, fol. 137vab.

\section{II. Éditions de textes}

Portugalia pontificia : Materials for the History of Portugal and the Papacy. 1198-1417, LINEHAN Peter (éd.), Lisboa, Fundação Calouste Gulbenkian, 2013.

ANSELMUS CANTUARIENSIS, De concordia praescientiae, praedestinationis et gratiae Dei cum libero arbitrio, Sancti Anselmi Opera Omnia, sснмітт Franciscus Salesius (éd.), Edinburgh, Nelson, 1940, vol. 2.

ANSELMUS CANTUARIENSIS, Epistola 313 (Ad Antonium), Sancti Anselmi Opera Omnia, SCHMITT Franciscus Salesius (éd.), Edinburgh, Nelson, 1940, vol. 5.

AUGUSTINUS HIPPONENSIS, Confessiones, MIGNE Jean-Paul (éd.), Patrologia Latina, 32, col. 720.

AUGUSTINUS HIPPONENSIS, De ciuitate Dei, MIGNE Jean-Paul (éd.), Patrologia Latina, 41, col. 599.

AUGUSTINUS HIPPONENSIS, Contra Faustum, MIGNE Jean-Paul (éd.), Patrologia Latina, 42, col. 249.

AVICENNA, Canon, Padoue, 1476.

BARTHOLOMAEUS ANGLICUS, De proprietatibus rerum, Liber XVI, Frankfurt, 1601.

BARTHOlomaeus ANGlicus, De proprietatibus rerum, vol. 1 : Prohemium, Libri I-IV, VAN DEN ABEele Baudouin, LONG R. James, MEYER Heinz, ROLING Bernd, TWOMEY Michael W. (éd.), « De diversis artibus, 78, n. s. 41 », Turnhout, Brepols, 2007a.

BARTHOLOMAEUS ANGLICUS, De proprietatibus rerum, vol. 6 : Liber XVII, VENTURA Iolanda (éd.), « De diversis artibus, 79, n. s. 42 », Turnhout, Brepols, $2007 \mathrm{~b}$.

BERNARDUS ClARAEVALLENSIS, A la louange de la Vierge Mère, huille Marie-Imelda et REGNARD Joël (éd.), « Sources Chrétiennes, 390 », Paris, 2009.

CASSIODORUS, Super psalmos, MIGNE Jean-Paul (éd.), Patrologia Latina, 70, col. 19.

HUGO DE SANCTO CARO, Postilla « Liber Apocalypsis », Venise, 1703.

IOHANNES AEGIDII ZAMORENSIS, El Prosodion de I. Aegidii Zemorensis : (un tratado gramatical hispano-latino inédito del s. XIII), ALONSO LóPEZ Luis (éd.), Barcelona, 1976 (thèse non publiée). IOHANNES AEGIDII ZAMORENSIS, Historia naturalis, GARCÍA BALLESTER Luis (éd.), Salamanca, Junta de Castilla y León, 1994.

IOHANNES AEGIDII ZAMORENSIS, Liber contra venena et animalia venenosa de Juan Gil de Zamora, FERRERO HERNÁNDEZ Cándida (éd.), Barcelona, Bellaterra Universitat Autònoma de Barcelona, 2009.

IOHANNES AEGIDII ZAMORENSIS, Sermonario. Juan Gil de Zamora. Estudio preliminar, edición, traducción y comentario de siete de sus sermones, LILLO REDONET F. (éd.) Zamora : Instituto de Estudios Zamoranos Florián de Ocampo, « Iohannis Aegidii Zamorensis Opera Omnia 1 », 2011. IOHANNES AEGIDII ZAMORENSIS, Una contribución al estudio de la prosa latina en Castilla del siglo XIII . La Edición crítica y estudio del Liber Mariae de Juan Gil de Zamora, BoHDZIEWICZ Soledad (éd.), Buenos Aires, 2014 (thèse non publiée, fUENTES Juan Hector dir.). 
IOHANNES AEGIDII ZAMORENSIS, Juan Gil de Zamora, Legende sanctorum et festiuitatum aliarum de quibus ecclesia sollempnizat. Leyendas de los santos y otras festividades que celebra la Iglesia. Introducción, edición crítica y traducción anotada, MARTín José Carlos (éd.) (OTERO PEREIRA Eduardo collab.), Zamora, Instituto de Estudios Zamoranos Florián de Ocampo, 2014. IOHANNES AEGIDII ZAMORENSIS, Breviloqvivm sermonvm virtvtvm et vitiorvm de Juan Gil de Zamora, estudio preliminar, editio princeps y traducción anotada, ATANASIO PERALTA Miguel Ángel (éd.), Salamanque, 2015 (thèse non publiée, MARTín José Carlos dir.), [disponible en ligne : https:// gredos.usal.es/jspui/bitstream/10366/128007/1/DFCI_AtanasioPeraltaM_EstudioPreli minar.pdf].

IOHANNES CHRYsostomus, Super Genesim, MIGNE Jean-Paul (éd.), Patrologia Graeca, 53, col. 175. IOHANNES DAmAscenus, De fide orthodoxa, MIGNE Jean-Paul (éd.), Patrologia Graeca, 94, col. 1175. IOHANNES DE SANCTO GEMINIANO, Liber de exemplis et similitudinibus rerum, Anvers, 1609. ISIDORUS HISPALENSIS, Etymologiae, MIGNE Jean-Paul (éd.), Patrologia Latina, 82, col. 585. MARCuS URBeVetensis, Marci de Urbi Veteri Liber de Moralitatibus, ETZKoRn Girard J. (éd.), New York, Franciscan Institute St. Bonaventure, 2005.

PASCHASIUS RADBERTUS, De Assumptione sanctae Mariae Virginis (uel Epistula beati Hieronymi ad Paulam et Eustochium de Assumptione), « Corpus Christianorum Continuatio Mediaevalis, 56C », RIPBERGER Albert (éd.), Turnhout, Brepols, 1985.

RUPERTUS TUITIENSIS, Commentaria in Canticum canticorum, « Corpus Christianorum Continuatio Mediaevalis, 26 », HAACKE Hraban (éd.), Turnhout, Brepols, 1974.

THOMAS CANTIMPRATENSIS, Liber de natura rerum, BOESE Helmut (éd.), Berlin - New York, Walter de Gruyter, 1973 (deux éditions sont indexées dans le corpus SourcEncyMe et utilisées dans cette étude : d'une part l'édition en cours en 2017 des versions I-II par Mattia CIPRIANI d'après sa thèse de doctorat soutenue en 2014, La place de Thomas de Cantimpré dans l'encyclopédisme médiéval : les sources du Liber de natura rerum - Tommaso di Cantimpré nell'enciclopedismo medievale : le fonti del Liber de natura rerum ; d'autre part l'édition provisoire de 1993 par Christian HÜNEMÖRDER et Konrad Benedikt VOLLMANN de la troisième version du De natura rerum réalisée dans le cadre du Projektgruppe B2 du SFB 226 Würzburg-Eichstätt - l'achèvement de cette édition du Thomas III, réalisé par Rudolf $\mathrm{K}$. Wiegand, est paru juste avant la mise en ligne de cet article : Thomas von Cantimpré, Liber de naturis rerum. Kritische Ausgabe der Redaktion III (Thomas III) eines Anonymus. Hrsg. vollmAnN, Benedikt Konrad, Déus, JANINE, Weigand, RUDolF KILIAN, Ulmschneider, HELGARD, Wiesbaden 2017 (Wissensliteratur im Mittelalter 54.1).

VINCENTIUS BELVACENSIS, Speculum naturale, version SM trifaria, Douai (éd.), 1624.

\section{Travaux}

VAN DEN ABEELE Baudouin, «L'allégorie animale dans les encyclopédies latines du Moyen Age », in BERLIOZ Jacques et POLO DE BEAULIEU Marie-Anne, (éd.), L'animal exemplaire au Moyen Age $\left(\mathrm{v}^{\mathrm{e}}-\mathrm{XV}^{\mathrm{e}}\right.$ siècles), Rennes, 1999, p. 123-143.

VAN DEN ABEELE Baudouin, « Moralisierte Enzyklopädien in der Nachfolge von Bartholomäus Anglicus : das 'Multifarium' in Wolfenbüttel und der 'Liber de exemplis et similitudinibus rerum' des Johannes de Sancto Geminiano ", in MEIER Christel, (éd.), Collection of Essays Die Enzyklopädie im Wandel vom Hochmittelalter bis zur frühen Neuzeit. Akten des Kolloquiums des Projekts D im Sonderforschungsbereich 231 (29.11. - 1.12.1996), München, 2002, p. 279-304. 
VAN DEN ABEELE Baudouin, Simbolismo sui margini. Le moralizzazioni del De proprietatibus rerum di Bartolomeo Anglico, in FARACI Dora (éd.), simbolismo animale e Letteratura, Roma, 2003, p. 159-183.

VAN DEN ABEELE Baudouin, « Diffusion et avatars d'une encyclopédie : le Liber de natura rerum de Thomas de Cantimpré ", in DE CALLATAŸ Godefroy et VAN DEN ABEELE Baudouin (éd.), Une lumière venue d'ailleurs. Héritages et ouvertures dans les encyclopédies d'Orient et d'Occident au Moyen Age Actes du colloque de Louvain-la-Neuve, 19-21 mai 2005, Turnhout, 2008, p. 141-176.

VAN DEN ABEELE Baudouin et MEYER Heinz «État de l'édition du 'De proprietatibus rerum' » in VAN DEN ABEELE Baudouin et MEYER Heinz (éd.), Bartholomaeus Anglicus, 'De proprietatibus rerum' : texte latin et réception vernaculaire : actes du colloque international, Münster, 9-11.10.2003, Turnhout, 2006, (De diversis artibus, 74), p. 11-12

VAN DEN ABEELE Baudouin, MEYER Heinz et RIBÉMONT Bernard, «Éditer l'encyclopédie de Barthélemy l'Anglais », Cahiers de recherches médiévales [En ligne], 6 | 1999a, mis en ligne le 28 mars 2008, consulté le 22 février 2017. URL : http://crm.revues.org/924. DOI : 10.4000/ crm.9241999

BATAILLON Louis-Jacques, « Les instruments de travail des prédicateurs au XIII ${ }^{\mathrm{e}}$ siècle », in HASENOHR Geneviève et LONGÈrE Jean (éd.), Culture et travail intellectuel dans l'Occident médiéval. Bilan des "Colloques d'humanisme médiéval" (1960-1980) fondés par le R.P. Hubert, O.P., Paris, 1981, p. 198-209.

BATAILLON Louis-Jacques, «Intermédiaires entre les traités de morale pratique et les sermons : les distinctiones bibliques alphabétiques », in BULTOT R. (éd.) Les Genres littéraires dans les sources théologiques et philosophiques médiévales, Turnhout, 1982, p. 213-226.

BERTRAND Paul et VAN DEN ABEELE Baudouin, « Recyclage de contenus et récupération de pièces d'archives dans le Macrologus encyclopédique de Saint-Laurent de Liège (circa 1470-1480) », in ClAASSENS Geert et VERBEKE Werner (éd.), Medieval Manuscripts in Transition. Tradition and Creative Recycling, Leuven, 2006, (Mediaevalia lovaniensia. Series 1, Studia), p. 37-59.

DAHAN Gilbert, " Encyclopédies et exégèse de la Bible aux XII ${ }^{\mathrm{e}}$ et XIII ${ }^{\mathrm{e}}$ siècles ", Cahiers de recherches médiévales [En ligne], 6 | 1999a, mis en ligne le 11 janvier 2007, consulté le 22 février 2017. URL : http://crm.revues.org/927. DOI : 10.4000/crm.927.

DAHAN Gilbert, L'Exégèse chrétienne de la Bible en Occident médiéval, Paris, 1999b.

DONDAINE Antoine, « La vie et les oeuvres de Jean de San Gimignano », Archivum Fratrum Praedicatorum, 9, p. 128-183.

DRAELANTS Isabelle, « La science naturelle et ses sources chez Barthélemy l'Anglais et les encyclopédistes contemporains ", in VAN DEN ABEELE Baudouin et MEYER Heinz (éd.),

Bartholomaeus Anglicus, "De proprietatibus rerum" : texte latin et réception vernaculaire : actes du colloque international, Münster, 9-11.10.2003, Turnhout, 2006, (De diversis artibus, 74), p. 43-99.

DRAELANTS Isabelle, «Scala mundi, scala celi de la A a la Z : claves para la comprensión de la obra universal de Juan Gil de Zamora. Exégesis, libri authentici y mediadores », Studia Zamorensia (segunda etapa), 13 [En ligne], 2014, mis en ligne en décembre 2014, consulté le 22 février 2017. URL : http://revistas.uned.es/index.php/studiazamo/article/view/13691/12384, DOI : http:// dx.doi.org/10.5944/studiazamo.vol.13.2014.13691, p. 27-70. 
FRIEDMAN John B., «Peacocks and Preachers : Analytic Technique in Marcus of Orvieto's Liber de moralitatibus, Vatican lat. MS 5935 », in CLARK Willene B. et MCMUNN Meredith T. (éd.), Beasts and Birds of the Middle Ages. The Bestiary and Its Legacy, Philadelphia, 1989, p. 179-196.

HAMY Adrienne, «Juan Gil de Zamora, Apis Dei : hallazgos homiléticos y propuestas », Studia Zamorensia (segunda etapa), 13 [En ligne], 2014, mis en ligne en décembre 2014, consulté le 22 février 2017. URL : http://revistas.uned.es/index.php/studiazamo/article/view/13694/12386. DOI : http://dx.doi.org/10.5944/studiazamo.vol.13.2014.13694, p. 71-93.

LONCKE Jérémy, «Approche comparative de la diffusion et de la transmission des différents états manuscrits du De Proprietatibus Rerum de Barthélemy l'Anglais », in DE CALLATAY Godefroy et VAN DEN ABEELE Baudouin (éd.), Une lumière venue d'ailleurs. Héritages et ouvertures dans les encyclopédies d'Orient et d'Occident au Moyen Age Actes du colloque de Louvain-la-Neuve, 19-21 mai 2005, Turnhout, 2008, p. 177-198.

LONGÈRE Jean, La Prédication médiévale, Paris, 1983.

MARTínez GÁzquez José, « Moralización de las piedras preciosas en la Historia Naturalis de Juan Gil de Zamora (1240-1320) », Homenaje a M. Balasch, Faventia, 20/2, 1998, p. 177-186.

MARTínEZ GÁzQUEZ José, « Moralización de los animales de Juan Gil de Zamora (s. XIII) », Micrologus, 8, 2000, p. 237-259.

MEIER Christel, « Text und Kontext. Steine und Farben bei Bartholomäus Anglicus in ihren Werkund Diskurszusammenhängen ", in VAN DEN ABEELE Baudouin et MEYER Heinz (éd.), Bartholomaeus Anglicus, "De proprietatibus rerum" : texte latin et réception vernaculaire : actes du colloque international, Münster, 9-11.10.2003, Turnhout, 2006, (De diversis artibus, 74), p. 151-184.

MEYER Heinz, « Zu Formen und Funktionen der Textbearbeitung und Werkerschließung in der Überlieferung des 'Liber de proprietatibus rerum' ", in MEIER Christel, HÜPPER Dagmar et KELLER Hagen (éd.), Der Codex im Gebrauch, München, 1996, p. 211-223.

MEYER Heinz, Die Enzyklopädie des Bartholomäus Anglicus. Untersuchungen zur Überlieferungsund Rezeptiongeschichte von « De proprietatibus rerum », (Münstersche Mittelalter-Schriften, 77), München, 2000.

OLDONI Massimo et ZAPPERI Andrea, Giovanni da San Gimignano. Un enciclopedico dell'anima. Studi e testi, San Gimignano, 1993.

PAULMIER-FOUCART Monique (DUCHENNE Marie-Christine collab.), Vincent de Beauvais et le Grand Miroir du monde, (Témoins de notre Histoire, 10), Turnhout, 2004.

PONS Philippe, «Les auto-références dans l'œuvre encyclopédique de Juan Gil de Zamora comme témoignage de la conception progressive de l'ouvrage ", Spicae, 2, 2012, p. 121-141.

ROUSE Richard H., « L'évolution des attitudes envers l'autorité écrite : le développement des instruments de travail au XIII ${ }^{\mathrm{e}}$ siècle ", in HASENOHR Geneviève et LONGÈRE Jean (éd.), Culture et travail intellectuel dans l'Occident médiéval. Bilan des "Colloques d'humanisme médiéval" (1960-1980) fondés par le R.P. Hubert, O.P., Paris, 1981, p. 115-144.

VECCHIo Silvana, « Giovanni da San Gimignano », Dizionario Biografico degli Italiani, vol. 56, Roma : Istituto della Enciclopedia italiana, 2001.

VENTURA Iolanda, « Die moralisierten Enzyklopädien des späteren Mittelalters : ein überblick unter Berücksichtigung der Fallbeispiele des 'Lumen Anime', des 'Liber de exemplis et similitudinibus rerum' und des 'Liber Similitudinum Naturalium' », Reti medievali, 4, janvier 2003/1. 
WENZEL Siegfried, Medieval Artes Praedicandi : A Synthesis of Scholastic Sermon Structure, Toronto, 2015.

\section{NOTES}

1. MARTíNEZ GÁZQUEZ J., « Moralización de las piedras preciosas en la Historia Naturalis de Juan Gil de Zamora (1240-1320)», Homenaje a M. Balasch, Faventia, 20/2, 1998, p. 177-186 et « Moralización de los animales de Juan Gil de Zamora (s. XIII) », Micrologus, 8, 2000, p. 237-259.

2. HAMY A., «Juan Gil de Zamora, Apis Dei : hallazgos homiléticos y propuestas », Studia Zamorensia (segunda etapa) [En ligne], 13 | 2014, p. 71-93.

3. IOHANNES AEGIDII ZAMORENSIS, Historia naturalis, GARCÍA BALLESTER L., Salamanca, 1994.

4. VAN DEN ABEele B., " Diffusion et avatars d'une encyclopédie : le Liber de natura rerum de Thomas de Cantimpré ", in DE CALLATAŸ G. et VAN DEN ABEELE B. (dir.), Une lumière venue d'ailleurs. Héritages et ouvertures dans les encyclopédies d'Orient et d'Occident au Moyen Age Actes du colloque de Louvain-laNeuve, 19-21 mai 2005, Turnhout, 2008, p. 141-176.

5. PAULMIER-FOUCART M., Vincent de Beauvais et le Grand Miroir du monde, « Témoins de notre histoire 10 », Turnhout, 2004 : 44-58, et comparaison avec les abeilles chez Juan Gil de Zamora : 338-341.

6. Sur cet auteur, notamment: D ONDAINE A. 1939 «La vie et les oeuvres de Jean de San Gimignano ", Archivum Fratrum Praedicatorum, vol. 9, p. 128-183; OLDONI M. et ZAPPERI A. Giovanni da San Gimignano. Un enciclopedico dell'anima. Studi e testi, San Gimignano, 1993 ; VAN DEN ABEELE B. «Moralisierte Enzyklopädien in der Nachfolge von Bartholomäus Anglicus : das 'Multifarium' in Wolfenbüttel und der 'Liber de exemplis et similitudinibus rerum' des Johannes de Sancto Geminiano ", in Collection of Essays Die Enzyklopädie im Wandel vom Hochmittelalter bis zur frühen Neuzeit. Akten des Kolloquiums des Projekts D im Sonderforschungsbereich 231 (29.11. - 1.12.1996), MEIER Ch. (dir.), München, 2002, p. 279-304; VECCHIO s. «Giovanni da San Gimignano », Dizionario Biografico degli Italiani, vol. 56, Roma : Istituto della Enciclopedia italiana, 2001 ; VENTURA I. « Die moralisierten Enzyklopädien des späteren Mittelalters : ein überblick unter Berücksichtigung der Fallbeispiele des 'Lumen Anime', des 'Liber de exemplis et similitudinibus rerum' und des 'Liber Similitudinum Naturalium' », Reti medievali, 4, janvier 2003/1. La populaire Summa de exemplis ac similitudinibus rerum perutilis praedicatoribus de ce frère prêcheur (1260/70 - c. 1333), en dix livres systématiquement organisés dans l'ordre alphabétique et explicitement destinés à fournir matière naturaliste aux sermons, fut probablement rédigée après 1298 et avant 1314 : l'univers entier y forme un immense répertoire allégorique. Le texte en est intégré dans la base de données SourcEncyMe de l'IRHT, à partir de l'édition d'Anvers de 1609. Jean aurait pris l'habit au couvent dominicain de Sienne puis étudié au studium de Barcelone. On le trouve au chapitre provincial de Pistoia en 1299 comme lecteur du couvent de Santa Maria sopra Minerva, puis dans divers actes de donation à l'occasion de la fondation d'un couvent dominicain à San Gimignano. Sa production est essentiellement homilétique en latin ou en vernaculaire (citons les recueils de tempore, de sanctis, de mortuis dont une partie ad status, un Quadragesimale, un De operibus sex dierum). Beatrice Amelotti rédige actuellement une thèse sur le livre Ix de sa Summa, consacré aux artes mechanicae.

7. Je remercie Isabelle Draelants, Nicole Bériou et Monique Paulmier-Foucart pour les stimulants et précieux conseils qui ont enrichi et structuré ma réflexion.

8. VAN DEN ABEELE B., "L'allégorie animale dans les encyclopédies latines du Moyen Age », in BERLIOZ J. et POLO DE BEAULIEU M.-A., L'animal exemplaire au Moyen Age ( $V^{e}-X V^{e}$ siècles), Rennes, 1999, p. 123-143: 123 .

9. BERTRAND P. et alii, «Recyclage de contenus et récupération de pièces d'archives dans le Macrologus encyclopédique de Saint-Laurent de Liège (circa 1470-1480)», in CLAASSENS G. et 
VERBEKE W. (dir.), Medieval manuscripts in transition. Tradition and creative recycling, Leuven, 2006, « Mediaevalia lovaniensia. Series 1, Studia », p. 37-59: 38.

10. VAN DEN ABEELE « Diffusion », $2008: 5$.

11. MEYER H., Die Enzyklopädie des Bartholomäus Anglicus. Untersuchungen zur Überlieferungs- und Rezeptiongeschichte von "De proprietatibus rerum », " Münstersche Mittelalter-Schriften, 77 ", München, 2000 ; VAN DEN ABEELE B., Simbolismo sui margini. Le moralizzazioni del De proprietatibus rerum di Bartolomeo Anglico, in FARACI D., simbolismo animale e Letteratura, Roma, 2003, p. 159-183 ; M EIER ch., « Text und Kontext. Steine und Farben bei Bartholomäus Anglicus in ihren Werk- und Diskurszusammenhängen ", in VAN DEN ABEELE B. et MEYER H. (dir.), Bartholomaeus Anglicus, "De proprietatibus rerum": texte latin et réception vernaculaire: actes du colloque international, Münster, 9-11.10.2003, Turnhout, 2006, «De diversis artibus, $74 »$, p. 151-184; LONCKE J., "Approche comparative de la diffusion et de la transmission des différents états manuscrits du De Proprietatibus Rerum de Barthélemy l'Anglais ", in DE CALLATAY G. et VAN DEN ABEELE B. (dir.), Une lumière venue d'ailleurs. Héritages et ouvertures dans les encyclopédies d'Orient et d'Occident au Moyen Age Actes du colloque de Louvain-la-Neuve, 19-21 mai 2005, Turnhout, 2008, p. 177-198.

12. J'achève actuellement une thèse de doctorat sur les sermons mariaux de cet auteur dont je propose une editio princeps.

13. DAHAN G., " Encyclopédies et exégèse de la Bible aux XII ${ }^{\mathrm{e}}$ et XIII ${ }^{\mathrm{e}}$ siècles ", Cahiers de recherches médiévales [En ligne], 6 | 1999a, mis en ligne le 11 janvier 2007, consulté le 22 février 2017. URL: http://crm.revues.org/927. DOI : 10.4000/crm.927, p. 24-33.

14. ROUSE R. H., «L'évolution des attitudes envers l'autorité écrite: le développement des instruments de travail au XIII ${ }^{\mathrm{e}}$ siècle ", in HASENOHR G. et LONGÈRE J. (dir.), Culture et travail intellectuel dans l'Occident médiéval. Bilan des "Colloques d'humanisme médiéval" (1960-1980) fondés par le R.P. Hubert, O.P., Paris, 1981, p. 115-144 ; BATAILLON L.-J., «Les instruments de travail des prédicateurs au XIII siècle ", in HASENOHR Geneviève et LONGÈRE J. (dir.), Culture et travail intellectuel dans l'Occident médiéval. Bilan des "Colloques d'humanisme médiéval" (1960-1980) fondés par le R.P. Hubert, O.P., Paris, 1981, p. 198-209.

15. DRAELANTS I., «La science naturelle et ses sources chez Barthélemy l'Anglais et les encyclopédistes contemporains ", in VAN DEN ABEELE B. et MEYER H. (dir.), Bartholomaeus Anglicus, "De proprietatibus rerum" : texte latin et réception vernaculaire : actes du colloque international, Münster, 9-11.10.2003, Turnhout, 2006, « De diversis artibus, 74 », p. 43-99: 45.

16. LINEHAN P., Portugalia pontificia: Materials for the History of Portugal and the Papacy. 1198-1417, Lisboa, 2013, vol. I, p. 536

17. MARCUS URBEVETENSIS, Marci de Urbi Veteri Liber de Moralitatibus, ETZKORN G. J., New York, 2005 : III ; FRIEDMAN J. B., « Peacocks and Preachers : Analytic Technique in Marcus of Orvieto's Liber de moralitatibus, Vatican lat. MS 5935 ", in CLARK W. B. et MCMUNN M. T. (dir.), Beasts and Birds of the Middle Ages. The Bestiary and Its Legacy, Philadelphia, 1989, p. 179-196: p. 183.

18. DRAELANTS, « La science », $2006: 47-48$.

19. VAN DEN ABEELE B. et alii: BARTHOLOMAEUS ANGLICUS, De proprietatibus rerum, vol. 1 : Prohemium, Libri I-IV, «De diversis artibus, 78, n. s. 41 », Turnhout, 2007a; BARTHOLOMAeUS ANGLICUS, De proprietatibus rerum, vol. 6: Liber XVII, VENTURA I., "De diversis artibus, 79, n. s. 42 », Turnhout, 2007b ; lib. VI, VIII et IX à paraître.

20. Seuls demeurent, en sus des entrées en $\mathrm{A}$, un index de la lettre $\mathrm{B}$ et des références à diverses entrées, sinon rédigées, du moins prévues. 
21. DRAELANTS I., "Scala mundi, scala celi de la A a la Z : claves para la comprensión de la obra universal de Juan Gil de Zamora. Exégesis, libri authentici y mediadores », Studia Zamorensia (segunda etapa), 13 [En ligne], 2014, p. 27-70.

22. PONS P., «Les auto-références dans l'œuvre encyclopédique de Juan Gil de Zamora comme témoignage de la conception progressive de l'ouvrage », Spicae, 2, 2012, p. 121-141

23. IOHANNES AEGIDII ZAMORENSIS: Archivus sive Armarium scripturarum inédit; Liber illustrium personarum inédit ; Prosodion: El Prosodion de I. Aegidii Zemorensis : (un tratado gramatical hispanolatino inédito del s. XIII), ALONSO LóPEZ L., Barcelona, 1976 (thèse non publiée); Liber Marie: Una contribución al estudio de la prosa latina en Castilla del siglo XIII. La Edición crítica y estudio del Liber Mariae de Juan Gil de Zamora, BoHDZIEWICZ S., Buenos Aires, 2014 (thèse, en cours de publication); Liber contra uenena: Liber contra venena et animalia venenosa de Juan Gil de Zamora, FERRERO HERNÁNDEZ C., Barcelona, 2009 ; Legende sanctorum: Juan Gil de Zamora, Legende sanctorum et festiuitatum aliarum de quibus ecclesia sollempnizat. Leyendas de los santos y otras festividades que celebra la Iglesia. Introducción, edición crítica y traducción anotada, MARTíN J. C., Zamora, 2014

24. BARTHOLOMAEUS ANGLICUS 2007a: 12 .

25. DAHAN G., L'Exégèse chrétienne de la Bible en Occident médiéval, Paris, 1999b : p. 134-151.

26. BATAILLON L.-J., «Intermédiaires entre les traités de morale pratique et les sermons : les distinctiones bibliques alphabétiques ", in BULTOT R. (éd.), Les Genres littéraires dans les sources théologiques et philosophiques médiévales, Turnhout, 1982, p. 213-226.

27. Les notices utilisées dans cette étude recourent à Barthélemy, sauf mentions contraires. Le cadre restreint de cette étude ne me permet pas d'examiner les rapports de l'HN et du LM avec le Speculum naturale de Vincent de Beauvais. Les mêmes contraintes empêchent une comparaison avec le petit corpus de cent-cinquante marginalia répartis avant tout dans les livres IV et $\mathrm{V}$ de Thomas de Cantimpré (VAN DEN ABEELE $2008: 156)$.

28. BARTHOLOMAEUS ANGLICUS : 2007 a.

29. DAHAN « Encyclopédies ", 1999a : 30.

30. BARTHOLOMAEUS ANGLICUS 2007a : 10-11.

31. MEYER H., « Zu Formen und Funktionen der Textbearbeitung und Werkerschließung in der Überlieferung des 'Liber de proprietatibus rerum' », in MEIER Ch., HÜPPER D. et KELLER H., Der Codex im Gebrauch, München, 1996, p. 211-223 : p. 213-214; MEYER : 2000 et son équipe de recherche: Seminar für mittellateinische Philologie de l'Université de Münster. Volume collectif issu d'un colloque tenu à Münster en 2003 : VAN DEN ABEELE B. et alii, Bartholomaeus Anglicus, 'De proprietatibus rerum': texte latin et réception vernaculaire: actes du colloque international, Münster, 9-11.10.2003, Turnhout, 2006, « De diversis artibus, $74 »$.

32. VAN DEN ABEELE « L'allégorie », $1999: 23$.

33. MEIER « Text », $2006: 179$.

34. À partir de la base de données modélisée par Jérémy Loncke et inachevée: http:// www.lejer.be/marginalia/index.htm. LONCKE 2008 : 179-180.

35. VINCENTIUS BELVACENSIS, Speculum naturale, version SM trifaria, éd. Douai, 1624, lib. XII, cap. XLIV ; THOMAS CANTIMPRATENSIS, Liber de natura rerum, version "Thomas III. ", éd. VOLLMAN B. K. HÜNEMÖRDER Ch., 1992, lib. XI, cap. III ; THOMAS CANTIMPRATENSIS, Liber de Natura rerum, version I-II, éd. CIPRIANI M. en cours, 2017, lib. X, cap. VII ; MARCUS URBEVETENSIS, LM 6.3 ; IOHANNES DE SANCTO GEMINIANO, LESR 3.51 .

36. IOHANNES DE SANCTO GEMINIANO, Liber de exemplis et similitudinibus rerum, éd. Anvers, 1609, lib. III, cap. LI : «Pauper spiritu assimilatur abieti. Primo, quia abies ipsa sublimiter augmentatur, unde secundum Isidorum dicitur abies ab eundo, eo quod pre ceteris arboribus in altum eundo porrigitur ». Texte accessible en ligne, d'après l'édition d'Anvers de 1609, sur le site 
SourcEncyMe, http://sourcencyme.irht.cnrs.fr/, hébergé actuellement par l'Institut de recherche et d'histoire des textes, Isabelle Draelants étant porteuse du projet.

37. MARCUS URBEVETENSIS LM 6.3 ; IOHANNES DE SANCTO GEMINIANO, LESR 3.51.

38. VINCENTIUS BELVACENSIS, SN 12.44; THOMAS CANTIMPRATENSIS, LNR III 11.3; THOMAS CANTIMPRATENSIS, LNR I-II 10.7 ; IOHANNES AEGIDII ZAMORENSIS HN 1.2 ; IOHANNES DE SANCTO GEMINIANO, LESR 3.51.

39. IOHANNES AEGIDII ZAMORENSIS HN 1.2 ; IOHANNES DE SANCTO GEMINIANO, LESR 3.51.

40. IOHANNES DE SANCTO GEMINIANO, LESR 3.51.

41. IOHANNES AEGIDII ZAMORENSIS HN 1.2 .

42. IOHANNES AEGIDII ZAMORENSIS HN 1.2 .

43. DRAELANTS « Scala », $2014: 52-53$.

44. WEnZEL S., Medieval Artes Praedicandi : A Synthesis of Scholastic Sermon Structure, Toronto, 2015 : 46-47.

45. PONS « Les auto-références », $2012: 121-141$.

46. J'en ai mis au jour différents témoins ces dernières années, en sus du Ms. Assise, Sacro Convento, 414 : Ms. Olomouc, Vědecká Knihovna, M.II.243 ; Ms. Cieszyn, Książnica Cieszyńska, DD-V-7: ces deux premiers sur les indications de Cándida Ferrero Hernández; Ms. Paris, Bibliothèque Nationale de France, Lat. 18195; Ms. Cambridge, Jesus College, Q.A.13; Ms. Worcester, Cathedral, F.126 ; Ms. Lisbonne, Biblioteca Nacional de Portugal, IL. 44. Cieszyn donne vingt-deux sermons du commun des saints, huit sermons ad status et douze sermons rituales; Cambridge livre trente-cinq sermones temporales; Lisbonne contient deux collections, l'une livre cinq sermons temporales et un sermon ritualis, l'autre cent-six sermons temporales; Olomouc cent trente-quatre sermons temporales, vingt collationes et trente méditations ; Paris quarante sermons temporales; Worcester seize sermons temporales.

47. Ms. Burgo de Osma, Archivo Catedralicio, $110\left(=\mathrm{O}^{110}\right)$ et Ms. Madrid, Biblioteca Nacional de España, 9503 (= M).

48. IOHANNES AEGIDII ZAMORENSIS : 2014.

49. Ms. Burgo de Osma, Archivo Catedralicio, 18 ; Ms. Florence, Biblioteca Laurenziana, Convento Soppresso, B.7.8 et Ms. Salamanque, Biblioteca Universitaria, 2081.

50. Sept des sermones temporales ont fait l'objet d'un travail de master désormais publié : IOHANNES AEGIDII ZAMORENSIS, Sermonario. Juan Gil de Zamora. Estudio preliminar, edición, traducción y comentario de siete de sus sermones, LILLO REDONET F. (éd.) Zamora : Instituto de Estudios Zamoranos Florián de Ocampo, «Iohannis Aegidii Zamorensis Opera Omnia 1 », 2011.

51. Dont je propose une editio princeps dans ma thèse de doctorat.

52. Je donne une editio princeps de l'un des deux sermons pro curiis magnatorum dans un article en cours de publication : «A Glance at the Music of Time : un sermon pro curiis magnatorum inédit de Juan Gil de Zamora (OFM, c.1290) ». Son thema est Diligite iustitiam.

53. IOHANNES AEGIDII ZAMORENSIS, Breviloqvivm sermonvm virtvtvm et vitiorvm de Juan Gil de Zamora, estudio preliminar, editio princeps y traducción anotada, ATANASIO PERALTA M. Á. (éd.), Salamanque, 2015 (thèse).

54. Je souligne le terme du lemme thématique ensuite développé dans chaque sermon marial, édité dans ma thèse de doctorat.

55. IOHANNES AEGIDII ZAMORENSIS, Breviloqvivm, ATANASIO éd., 2015 : 143 (thèse).

56. Sauf mentions contraires.

57. Source non relevée par ETZKORN qui précise ne pas avoir trouvé la citation dans le Platearius dans l'édition 1497 de Venise. 
58. Source non relevée par ETZKORN qui précise ne pas avoir trouvé la citation dans le Lapidarius de Marbode. Isabelle Draelants, que je remercie, m'indique que Barthélemy utilise sous «Lapidarius » une version de Marbode interpolée avec les lettres d'Evax et le lapidaire classé alphabétiquement de Damigéron-Evax à l'époque de Constantin l'Africain.

59. On retrouve cette citation, sans mention du Lapidaire et appliquée au prédicateur, dans la Summa plus tardive de Jean de San Gimignano, lib. II, cap. XXVII : "Item argentum est balsami conservativum, et virtutis in iaspide custoditivum. Unde maiorem virtutem habet iaspis in annulo argenteo, quam aureo » (éd. Anvers, 1609).

60. Source non trouvée par ETZKORN.

\section{RÉSUMÉS}

L'activité de prédication au XIII ${ }^{\mathrm{e}} \mathrm{s}$. oriente la confection d'encyclopédies par les frères mendiants. Chez Juan Gil de Zamora, polygraphe franciscain ibérique de la seconde moitié du XIII ${ }^{\mathrm{e}}$ siècle, les rapports entre prédication et savoir encyclopédique sont particulièrement étroits. Ses sermons empruntent à la Nature thema et exempla, ce qui est commun. Mais, de manière plus étonnante, son encyclopédie naturelle s'adresse aux prédicateurs au point de leur fournir des ressources pour leurs sermons, et même des sermons thématiques intégraux appuyés sur des moralisations de certaines propriétés naturelles. Est-ce un procédé original ? L'Historia naturalis de Juan Gil de Zamora est ici comparée au Liber de moralitatibus de Marc d'Orvieto, le plus proche de sa démarche. Ces deux ouvrages seront confrontés aux marginalia de Barthélemy l'Anglais et à des sermons inédits de l'Opus sermonum copiosum et du Liber Marie de Juan Gil de Zamora.

Preaching activity influences the production of encyclopedia by the mendicant friars from the thirteenth century onwards. A Franciscan and Iberian polygraph, Juan Gil of Zamora strikingly uses these connections between encyclopedia and preaching in his works. Commonly enough, his sermons take thema and exempla from Nature. However, Juan Gil de Zamora's Historia naturalis displays a full range of homiletic tools for preachers, indeed complete thematic sermons as res naturales moralizations. Is this unusual ? Marcus of Orvieto's Liber de moralitatibus seems to take the closest path. And both the Liber and the Historia will be compared to Bartholomaeus Anglicus' marginalia and to some Gil of Zamora's unpublished sermons, included in his Liber Marie and Opus sermonum copiosum.

\section{INDEX}

Mots-clés : Encyclopedia, Moralization, Homiletics, Marginalia, Franciscans

Keywords : Encyclopédie, moralisation, homilétique, marginalia, Franciscains 


\section{AUTEUR}

\section{ADRIENNE HAMY-DUPONT}

Adrienne DUPONT HAMY (EPHE - ED 472 - Langue, Littérature, Image : Civilisation et Sciences Humaines - ED 131), agrégée de lettres classiques, vient d'achever sous la direction de J.-Fr. Cottier (Paris VII) et de P. Henriet (EPHE) une thèse de doctorat intitulée : « Système encyclopédique et prédication mariale chez Juan Gil de Zamora (OFM, + c. 1306). Les sermones virginales : édition, traduction et commentaire ». 